Supporting information

\title{
High coordinate metal iodate chlorides with diverse structural motifs and tunable birefringence
}

Ying-Jie $\mathrm{Jia}^{\dagger}$, Yi-Gang Chen ${ }^{\dagger}$, Ting-Wang ${ }^{\dagger}$, Yao Guo ${ }^{\ddagger}$, Xiao-Fang Guan ${ }^{\dagger}$, Xian-Ming Zhang ${ }^{\dagger} *$

${ }^{\dagger}$ Key Laboratory of Magnetic Molecules and Magnetic Information Material of Ministry of Education, School of Chemistry and Material Science, Shanxi Normal University, Linfen 041004, China

${ }^{\ddagger}$ Henan Joint International Research Laboratory of Nanocomposite Sensing Materials, School of Chemical and Environmental Engineering, Anyang Institute of Technology, Anyang 455000, China 
Table S1. Selected bond distances $(\AA)$ for for compounds $\mathbf{1}-\mathbf{5}$.

Table S2. Anisotropic displacement parameters $\left(\AA^{2} \times 10^{3}\right)$ for compounds $\mathbf{1}-\mathbf{5}$.

Table S3. The birefringence of metal iodates without other large polarizability anisotropy groups.

Figure S1. Experimental and simulated PXRD patterns of 1-5.

Figure S2. Energy-dispersive spectrometry (EDS) plots of 1-5.

Figure S3. Asymmetric unit of $\mathrm{Ba}\left(\mathrm{IO}_{3}\right) \mathrm{Cl}$.

Figure S4. Asymmetric unit of $\mathrm{Ba}\left(\mathrm{IO}_{3}\right) \mathrm{Cl}\left(\mathrm{H}_{2} \mathrm{O}\right)$.

Figure S5. Asymmetric unit of $\mathrm{Ba}_{7}\left(\mathrm{IO}_{3}\right)_{5} \mathrm{Cl}_{9}$.

Figure S6. Asymmetric unit of $\mathrm{La}\left(\mathrm{IO}_{3}\right)_{2} \mathrm{Cl}$.

Figure S7. Asymmetric unit of $\mathrm{La}_{2}\left(\mathrm{IO}_{3}\right)_{5} \mathrm{Cl}$.

Figure S8. TG-DTA curves of $\mathrm{Ba}\left(\mathrm{IO}_{3}\right) \mathrm{Cl}(\mathbf{a}), \mathrm{Ba}\left(\mathrm{IO}_{3}\right) \mathrm{Cl}\left(\mathrm{H}_{2} \mathrm{O}\right)(\mathbf{b}), \mathrm{Ba}_{7}\left(\mathrm{IO}_{3}\right)_{5} \mathrm{Cl}_{9}$ (c), $\mathrm{La}\left(\mathrm{IO}_{3}\right)_{2} \mathrm{Cl}(\mathbf{d})$ and $\mathrm{La}_{2}\left(\mathrm{IO}_{3}\right)_{5} \mathrm{Cl}(\mathbf{e})$.

Figure S9. The IR spectra of $\mathrm{Ba}\left(\mathrm{IO}_{3}\right) \mathrm{Cl}, \mathrm{Ba}\left(\mathrm{IO}_{3}\right) \mathrm{Cl}\left(\mathrm{H}_{2} \mathrm{O}\right), \mathrm{Ba}_{7}\left(\mathrm{IO}_{3}\right)_{5} \mathrm{Cl} 9, \mathrm{La}\left(\mathrm{IO}_{3}\right)_{2} \mathrm{Cl}$ and $\mathrm{La}_{2}\left(\mathrm{IO}_{3}\right)_{5} \mathrm{Cl}$.

Figure S10. UV-vis-IR spectra of $\mathrm{Ba}\left(\mathrm{IO}_{3}\right) \mathrm{Cl}, \mathrm{Ba}\left(\mathrm{IO}_{3}\right) \mathrm{Cl}\left(\mathrm{H}_{2} \mathrm{O}\right), \mathrm{Ba}_{7}\left(\mathrm{IO}_{3}\right)_{5} \mathrm{Cl}_{9}$, $\mathrm{La}\left(\mathrm{IO}_{3}\right)_{2} \mathrm{Cl}$ and $\mathrm{La}_{2}\left(\mathrm{IO}_{3}\right)_{5} \mathrm{Cl}$.

Figure S11. Calculated band structures, total and partial density of states (DOSs) of $\mathrm{Ba}\left(\mathrm{IO}_{3}\right) \mathrm{Cl}\left(\mathrm{H}_{2} \mathrm{O}\right)(\mathbf{a}), \mathrm{Ba}_{7}\left(\mathrm{IO}_{3}\right)_{5} \mathrm{Cl}_{9}(\mathbf{b})$ and $\mathrm{La}_{2}\left(\mathrm{IO}_{3}\right)_{5} \mathrm{Cl}$ (c).

Figure S12. Distinct arrangement of the $\mathrm{IO}_{3}$ groups in $\mathrm{Ba}\left(\mathrm{IO}_{3}\right) \mathrm{Cl}$ (a), $\mathrm{Ba}\left(\mathrm{IO}_{3}\right) \mathrm{Cl}\left(\mathrm{H}_{2} \mathrm{O}\right)(\mathbf{b}), \mathrm{Ba}_{7}\left(\mathrm{IO}_{3}\right)_{5} \mathrm{Cl}_{9}(\mathbf{c}), \mathrm{La}\left(\mathrm{IO}_{3}\right)_{2} \mathrm{Cl}(\mathbf{d})$ and $\mathrm{La}_{2}\left(\mathrm{IO}_{3}\right)_{5} \mathrm{Cl}(\mathbf{e})$. 
Table S1. Selected bond distances ( $\AA$ ) for compounds 1-5.

\begin{tabular}{|c|c|c|c|c|c|}
\hline \multicolumn{6}{|c|}{$\mathrm{Ba}\left(\mathrm{IO}_{3}\right) \mathrm{Cl}$} \\
\hline $\mathrm{Ba}(1)-\mathrm{O}(1)$ & $2.717(3)$ & $\mathrm{Ba}(1)-\mathrm{O}(1) \# 5$ & $3.1270(15)$ & $\mathrm{Ba}(1)-\mathrm{Cl}(1) \# 8$ & $3.5640(8)$ \\
\hline $\mathrm{Ba}(1)-\mathrm{O}(2) \# 1$ & $2.835(2)$ & $\mathrm{Ba}(1)-\mathrm{O}(1) \# 2$ & $3.1270(14)$ & $\mathrm{I}(1)-\mathrm{O}(1)$ & $1.803(3)$ \\
\hline $\mathrm{Ba}(1)-\mathrm{O}(2) \# 2$ & $2.835(2)$ & $\mathrm{Ba}(1)-\mathrm{Cl}(1)$ & $3.2575(12)$ & $\mathrm{I}(1)-\mathrm{O}(2)$ & $1.811(2)$ \\
\hline $\mathrm{Ba}(1)-\mathrm{O}(2) \# 3$ & $2.896(2)$ & $\mathrm{Ba}(1)-\mathrm{Cl}(1) \# 6$ & $3.2805(13)$ & $\mathrm{I}(1)-\mathrm{O}(2) \# 9$ & $1.811(2)$ \\
\hline $\mathrm{Ba}(1)-\mathrm{O}(2) \# 4$ & $2.896(2)$ & $\mathrm{Ba}(1)-\mathrm{Cl}(1) \# 7$ & $3.5640(8)$ & & \\
\hline \multicolumn{6}{|c|}{$\mathrm{Ba}\left(\mathrm{IO}_{3}\right) \mathrm{Cl}\left(\mathrm{H}_{2} \mathrm{O}\right)$} \\
\hline $\mathrm{Ba}(1)-\mathrm{O}(2)$ & $2.765(4)$ & $\mathrm{Ba}(1)-\mathrm{O}(4 \mathrm{~W}) \# 4$ & $2.949(4)$ & $\mathrm{I}(1)-\mathrm{O}(3)$ & $1.804(4)$ \\
\hline $\mathrm{Ba}(1)-\mathrm{O}(1) \# 1$ & $2.774(4)$ & $\mathrm{Ba}(1)-\mathrm{O}(3) \# 3$ & $3.000(4)$ & $\mathrm{I}(1)-\mathrm{O}(2)$ & $1.804(4)$ \\
\hline $\mathrm{Ba}(1)-\mathrm{O}(3) \# 2$ & $2.864(4)$ & $\mathrm{Ba}(1)-\mathrm{Cl}(1) \# 4$ & $3.2143(14)$ & $\mathrm{I}(1)-\mathrm{O}(1)$ & $1.818(4)$ \\
\hline $\mathrm{Ba}(1)-\mathrm{O}(2) \# 3$ & $2.887(4)$ & $\mathrm{Ba}(1)-\mathrm{Cl}(1) \# 1$ & $3.2300(14)$ & & \\
\hline $\mathrm{Ba}(1)-\mathrm{O}(1 \mathrm{~W})$ & $2.922(5)$ & $\mathrm{Ba}(1)-\mathrm{Cl}(1)$ & $3.3336(14)$ & & \\
\hline \multicolumn{6}{|c|}{$\mathrm{Ba}_{7}\left(\mathrm{IO}_{3}\right)_{5} \mathrm{Cl}_{9}$} \\
\hline $\mathrm{Ba}(1)-\mathrm{O}(2)$ & $2.677(3)$ & $\mathrm{Ba}(1)-\mathrm{Cl}(2) \# 1$ & $3.3956(11)$ & $\mathrm{Ba}(2)-\mathrm{Cl}(2)$ & 3.3011(11) \\
\hline $\mathrm{Ba}(1)-\mathrm{O}(2) \# 1$ & $2.822(3)$ & $\mathrm{Ba}(2)-\mathrm{O}(3)$ & $2.751(4)$ & $\mathrm{Ba}(2)-\mathrm{Cl}(2) \# 8$ & 3.3011(11) \\
\hline $\mathrm{Ba}(1)-\mathrm{O}(3) \# 1$ & $2.856(2)$ & $\mathrm{Ba}(2)-\mathrm{O}(3) \# 4$ & $2.751(4)$ & $\mathrm{I}(1)-\mathrm{O}(1) \# 9$ & $1.809(3)$ \\
\hline $\mathrm{Ba}(1)-\mathrm{O}(1)$ & $2.864(3)$ & $\mathrm{Ba}(2)-\mathrm{O}(3) \# 6$ & $2.751(4)$ & $\mathrm{I}(1)-\mathrm{O}(1)$ & $1.809(3)$ \\
\hline $\mathrm{Ba}(1)-\mathrm{O}(1) \# 2$ & $2.865(3)$ & $\mathrm{Ba}(2)-\mathrm{Cl}(2) \# 6$ & 3.3011(11) & $\mathrm{I}(1)-\mathrm{O}(1) \# 3$ & $1.809(3)$ \\
\hline $\mathrm{Ba}(1)-\mathrm{Cl}(2) \# 3$ & $3.1558(11)$ & $\mathrm{Ba}(2)-\mathrm{Cl}(2) \# 7$ & 3.3011(11) & $\mathrm{I}(2)-\mathrm{O}(2) \# 5$ & $1.806(3)$ \\
\hline $\mathrm{Ba}(1)-\mathrm{Cl}(2) \# 2$ & $3.2245(11)$ & $\mathrm{Ba}(2)-\mathrm{Cl}(2) \# 5$ & 3.3011(11) & $\mathrm{I}(2)-\mathrm{O}(2)$ & $1.806(3)$ \\
\hline $\mathrm{Ba}(1)-\mathrm{Cl}(1)$ & $3.2883(10)$ & $\mathrm{Ba}(2)-\mathrm{Cl}(2) \# 4$ & $3.3011(11)$ & $\mathrm{I}(2)-\mathrm{O}(3)$ & $1.836(4)$ \\
\hline \multicolumn{6}{|c|}{$\mathrm{La}\left(\mathrm{IO}_{3}\right)_{2} \mathrm{Cl}$} \\
\hline $\mathrm{La}(1)-\mathrm{O}(7) \# 2$ & $2.483(4)$ & $\mathrm{La}(2)-\mathrm{O}(9)$ & $2.413(4)$ & $\mathrm{I}(1)-\mathrm{O}(9) \# 5$ & $1.781(4)$ \\
\hline $\mathrm{La}(1)-\mathrm{O}(7)$ & $2.483(4)$ & $\mathrm{La}(2)-\mathrm{O}(8)$ & $2.438(4)$ & $\mathrm{I}(1)-\mathrm{O}(1)$ & $1.805(4)$ \\
\hline $\mathrm{La}(1)-\mathrm{O}(5)$ & $2.559(4)$ & $\mathrm{La}(2)-\mathrm{O}(2)$ & $2.585(4)$ & $\mathrm{I}(1)-\mathrm{O}(2)$ & $1.835(4)$ \\
\hline $\mathrm{La}(1)-\mathrm{O}(5) \# 2$ & $2.559(4)$ & $\mathrm{La}(2)-\mathrm{O}(5)$ & $2.626(4)$ & $\mathrm{I}(2)-\mathrm{O}(8) \# 3$ & $1.793(4)$ \\
\hline $\mathrm{La}(1)-\mathrm{O}(3) \# 2$ & $2.577(4)$ & $\mathrm{La}(2)-\mathrm{O}(4)$ & $2.632(4)$ & $\mathrm{I}(2)-\mathrm{O}(3)$ & $1.820(4)$ \\
\hline $\mathrm{La}(1)-\mathrm{O}(3)$ & $2.577(4)$ & $\mathrm{La}(2)-\mathrm{O}(6)$ & $2.721(4)$ & $\mathrm{I}(2)-\mathrm{O}(4)$ & $1.834(4)$ \\
\hline
\end{tabular}




\begin{tabular}{|l|l|l|l|l|l|}
\hline $\mathrm{La}(1)-\mathrm{O}(1)$ & $2.716(4)$ & $\mathrm{La}(2)-\mathrm{Cl}(1) \# 1$ & $2.8742(14)$ & $\mathrm{I}(3)-\mathrm{O}(7) \# 4$ & $1.793(4)$ \\
\hline $\mathrm{La}(1)-\mathrm{O}(1) \# 2$ & $2.716(4)$ & $\mathrm{La}(2)-\mathrm{Cl}(1)$ & $2.9962(14)$ & $\mathrm{I}(3)-\mathrm{O}(5)$ & $1.811(4)$ \\
\hline $\mathrm{La}(1)-\mathrm{O}(2) \# 2$ & $2.725(4)$ & $\mathrm{La}(2)-\mathrm{Cl}(2)$ & $3.0625(3)$ & $\mathrm{I}(3)-\mathrm{O}(6)$ & $1.826(4)$ \\
\hline $\mathrm{La}(1)-\mathrm{O}(2)$ & $2.725(4)$ & & & & \\
\hline \multicolumn{5}{|c|}{$\mathbf{L a}_{2}\left(\mathrm{IO}_{3}\right)_{5} \mathrm{Cl}$} \\
\hline $\mathrm{La}(1)-\mathrm{O}(2)$ & $2.428(5)$ & $\mathrm{La}(2)-\mathrm{O}(11)$ & $2.539(5)$ & $\mathrm{I}(2)-\mathrm{O}(9)$ & $1.823(4)$ \\
\hline $\mathrm{La}(1)-\mathrm{O}(8) \# 1$ & $2.468(5)$ & $\mathrm{La}(2)-\mathrm{O}(9)$ & $2.566(5)$ & $\mathrm{I}(2)-\mathrm{O}(7)$ & $1.827(4)$ \\
\hline $\mathrm{La}(1)-\mathrm{O}(4)$ & $2.467(5)$ & $\mathrm{La}(2)-\mathrm{O}(12)$ & $2.570(5)$ & $\mathrm{I}(3)-\mathrm{O}(1) \# 8$ & $1.801(5)$ \\
\hline $\mathrm{La}(1)-\mathrm{O}(1)$ & $2.486(5)$ & $\mathrm{La}(2)-\mathrm{O}(14)$ & $2.578(4)$ & $\mathrm{I}(3)-\mathrm{O}(3) \# 5$ & $1.810(5)$ \\
\hline $\mathrm{La}(1)-\mathrm{O}(7)$ & $2.507(5)$ & $\mathrm{La}(2)-\mathrm{O}(5) \# 5$ & $2.588(5)$ & $\mathrm{I}(3)-\mathrm{O}(10)$ & $1.826(5)$ \\
\hline $\mathrm{La}(1)-\mathrm{O}(7) \# 2$ & $2.639(4)$ & $\mathrm{La}(2)-\mathrm{O}(10)$ & $2.687(5)$ & $\mathrm{I}(4)-\mathrm{O}(2) \# 6$ & $1.794(5)$ \\
\hline $\mathrm{La}(1)-\mathrm{O}(15) \# 3$ & $2.669(5)$ & $\mathrm{La}(2)-\mathrm{Cl}(1)$ & $3.0084(16)$ & $\mathrm{I}(4)-\mathrm{O}(15)$ & $1.806(5)$ \\
\hline $\mathrm{La}(1)-\mathrm{O}(3)$ & $2.703(5)$ & $\mathrm{I}(1)-\mathrm{O}(6)$ & $1.788(5)$ & $\mathrm{I}(4)-\mathrm{O}(14)$ & $1.823(4)$ \\
\hline $\mathrm{La}(1)-\mathrm{O}(8) \# 2$ & $2.769(5)$ & $\mathrm{I}(1)-\mathrm{O}(5)$ & $1.795(5)$ & $\mathrm{I}(5)-\mathrm{O}(12)$ & $1.788(4)$ \\
\hline $\mathrm{La}(2)-\mathrm{O}(6)$ & $2.486(5)$ & $\mathrm{I}(1)-\mathrm{O}(4)$ & $1.814(5)$ & $\mathrm{I}(5)-\mathrm{O}(13)$ & $1.806(5)$ \\
\hline $\mathrm{La}(2)-\mathrm{O}(13) \# 4$ & $2.506(5)$ & $\mathrm{I}(2)-\mathrm{O}(8)$ & $1.809(4)$ & $\mathrm{I}(5)-\mathrm{O}(11) \# 7$ & $1.820(5)$ \\
\hline
\end{tabular}

Symmetry transformations used to generate equivalent atoms:

$\mathrm{Ba}\left(\mathrm{IO}_{3}\right) \mathrm{Cl}$ :

$\# 1-\mathrm{x}+1 / 2, \mathrm{y}+1 / 2, \mathrm{z}+1 / 2 ; \quad \# 2-\mathrm{x}+1 / 2,-\mathrm{y}+1, \mathrm{z}+1 / 2 ; \quad \# 3 \mathrm{x},-\mathrm{y}+3 / 2, \mathrm{z}+1 ; \quad \# 4 \mathrm{x}, \mathrm{y}, \mathrm{z}+1 ;$

$\# 5-\mathrm{x}+1 / 2,-\mathrm{y}+2, \mathrm{z}+1 / 2 ; \quad \# 6 \mathrm{x}-1 / 2, \mathrm{y},-\mathrm{z}+3 / 2 ; \quad \# 7-\mathrm{x}+1 / 2,-\mathrm{y}+1, \mathrm{z}-1 / 2 ;$

$\# 8-x+1 / 2,-y+2, z-1 / 2 ; \quad \# 9 x,-y+3 / 2, z$

$\mathrm{Ba}\left(\mathrm{IO}_{3}\right) \mathrm{Cl} \cdot \mathrm{H}_{2} \mathrm{O}$

$\# 1 \mathrm{x},-\mathrm{y}+1 / 2, \mathrm{z}-1 / 2 ; \quad \# 2-\mathrm{x}+1, \mathrm{y}-1 / 2,-\mathrm{z}+3 / 2 ; \quad \# 3-\mathrm{x}+1,-\mathrm{y}+1,-\mathrm{z}+1 ; \quad \# 4-\mathrm{x},-\mathrm{y}+1,-\mathrm{z}+1$

$\mathrm{Ba}_{7}\left(\mathrm{IO}_{3}\right)_{5} \mathrm{Cl}_{9}$

$\# 1-\mathrm{x}+\mathrm{y}+1,-\mathrm{x}+1, \mathrm{z} ; \quad \# 2-\mathrm{x}+2,-\mathrm{y}+1,-\mathrm{z}+1 ; \quad \# 3-\mathrm{y}+2, \mathrm{x}-\mathrm{y}, \mathrm{z} ; \quad \# 4-\mathrm{x}+\mathrm{y}+1,-\mathrm{x}+2, \mathrm{z} ;$

$\# 5 \mathrm{x}, \mathrm{y},-\mathrm{z}+1 / 2 ; \quad \# 6-\mathrm{y}+2, \mathrm{x}-\mathrm{y}+1, \mathrm{z} ; \quad \# 7-\mathrm{x}+\mathrm{y}+1,-\mathrm{x}+2,-\mathrm{z}+1 / 2 ;$

$\# 8-\mathrm{y}+2, \mathrm{x}-\mathrm{y}+1,-\mathrm{z}+1 / 2 ; \quad \# 9-\mathrm{x}+\mathrm{y}+2,-\mathrm{x}+2, \mathrm{z}$

$\mathrm{La}\left(\mathrm{IO}_{3}\right)_{2} \mathrm{Cl}$

$\# 1-\mathrm{x}+3 / 2,-\mathrm{y}+3 / 2,-\mathrm{z}+1 ; \quad \# 2-\mathrm{x}+1, \mathrm{y},-\mathrm{z}+3 / 2 ; \quad \# 3 \mathrm{x}-1 / 2, \mathrm{y}+1 / 2, \mathrm{z} ;$

$\# 4-\mathrm{x}+3 / 2, \mathrm{y}+1 / 2,-\mathrm{z}+3 / 2 ; \quad \# 5 \mathrm{x}-1 / 2, \mathrm{y}-1 / 2, \mathrm{z}$

$\mathrm{La}_{2}\left(\mathrm{IO}_{3}\right)_{5} \mathrm{Cl}$

$\# 1 \mathrm{x}, \mathrm{y}+1, \mathrm{z} ; \quad \# 2-\mathrm{x}, \mathrm{y}+1 / 2,-\mathrm{z}+1 / 2 ; \quad \# 3 \mathrm{x},-\mathrm{y}+5 / 2, \mathrm{z}-1 / 2 ; \quad \# 4 \mathrm{x},-\mathrm{y}+3 / 2, \mathrm{z}-1 / 2$;

\#5 $\mathrm{x}, \mathrm{y}-1, \mathrm{z} ; \quad \# 6-\mathrm{x},-\mathrm{y}+2,-\mathrm{z}+1 ; \quad \# 7-\mathrm{x}+1, \mathrm{y}+1 / 2,-\mathrm{z}+3 / 2 ; \quad \# 8 \mathrm{x},-\mathrm{y}+3 / 2, \mathrm{z}+1 / 2$ 
Table S2. Anisotropic displacement parameters $\left(\AA^{2} \times 10^{3}\right)$ for compounds $\mathbf{1}-\mathbf{5}$.

The anisotropic displacement factor exponent takes the form: $-2 \pi^{2}\left[h^{2} a^{* 2} U_{11}+2 h k a * b * U_{12}\right]$.

\section{$\mathrm{Ba}\left(\mathrm{IO}_{3}\right) \mathrm{Cl}$}

\begin{tabular}{lllllll}
\hline & $\mathrm{U} 11$ & $\mathrm{U} 22$ & $\mathrm{U} 33$ & $\mathrm{U} 23$ & $\mathrm{U} 13$ & $\mathrm{U} 12$ \\
\hline $\mathrm{Ba}(1)$ & $12(1)$ & $20(1)$ & $11(1)$ & 0 & $2(1)$ & 0 \\
$\mathrm{I}(1)$ & $9(1)$ & $12(1)$ & $10(1)$ & 0 & $0(1)$ & 0 \\
$\mathrm{Cl}(1)$ & $19(1)$ & $38(1)$ & $29(1)$ & 0 & $-5(1)$ & 0 \\
$\mathrm{O}(1)$ & $14(2)$ & $30(2)$ & $16(1)$ & 0 & $-5(1)$ & 0 \\
$\mathrm{O}(2)$ & $18(1)$ & $13(1)$ & $17(1)$ & $-4(1)$ & $1(1)$ & $1(1)$ \\
\hline
\end{tabular}

$\mathrm{Ba}\left(\mathrm{IO}_{3}\right) \mathrm{Cl}\left(\mathrm{H}_{2} \mathrm{O}\right)$

\begin{tabular}{lllllll}
\hline & $\mathrm{U} 11$ & $\mathrm{U} 22$ & $\mathrm{U} 33$ & $\mathrm{U} 23$ & $\mathrm{U} 13$ & $\mathrm{U} 12$ \\
\hline $\mathrm{Ba}(1)$ & $15(1)$ & $16(1)$ & $16(1)$ & $1(1)$ & $2(1)$ & $2(1)$ \\
$\mathrm{I}(1)$ & $15(1)$ & $11(1)$ & $14(1)$ & $0(1)$ & $2(1)$ & $-1(1)$ \\
$\mathrm{Cl}(1)$ & $20(1)$ & $22(1)$ & $21(1)$ & $0(1)$ & $1(1)$ & $4(1)$ \\
$\mathrm{O}(1)$ & $27(2)$ & $16(2)$ & $23(2)$ & $6(2)$ & $3(2)$ & $-3(2)$ \\
$\mathrm{O}(2)$ & $24(2)$ & $20(2)$ & $15(2)$ & $-3(2)$ & $3(2)$ & $-4(2)$ \\
$\mathrm{O}(3)$ & $17(2)$ & $20(2)$ & $23(2)$ & $-2(2)$ & $8(2)$ & $-5(2)$ \\
$\mathrm{O}(4 \mathrm{~W})$ & $32(3)$ & $31(2)$ & $22(2)$ & $7(2)$ & $0(2)$ & $-6(2)$ \\
\hline
\end{tabular}

$\mathrm{Ba}_{7}\left(\mathrm{IO}_{3}\right)_{5} \mathrm{Cl}_{9}$

\begin{tabular}{lllllll}
\hline & $\mathrm{U} 11$ & $\mathrm{U} 22$ & $\mathrm{U} 33$ & $\mathrm{U} 23$ & $\mathrm{U} 13$ & $\mathrm{U} 12$ \\
\hline $\mathrm{Ba}(1)$ & $11(1)$ & $10(1)$ & $13(1)$ & $-1(1)$ & $-1(1)$ & $6(1)$ \\
$\mathrm{I}(2)$ & $8(1)$ & $9(1)$ & $12(1)$ & 0 & 0 & $5(1)$ \\
$\mathrm{Ba}(2)$ & $11(1)$ & $11(1)$ & $18(1)$ & 0 & 0 & $5(1)$ \\
$\mathrm{I}(1)$ & $9(1)$ & $9(1)$ & $9(1)$ & 0 & 0 & $5(1)$ \\
$\mathrm{Cl}(1)$ & $14(1)$ & $14(1)$ & $18(1)$ & 0 & 0 & $7(1)$ \\
$\mathrm{Cl}(2)$ & $14(1)$ & $19(1)$ & $17(1)$ & $-1(1)$ & $-2(1)$ & $5(1)$ \\
$\mathrm{O}(3)$ & $13(2)$ & $13(2)$ & $15(2)$ & 0 & 0 & $10(2)$ \\
$\mathrm{O}(2)$ & $18(2)$ & $16(2)$ & $18(2)$ & $6(1)$ & $6(1)$ & $11(1)$ \\
$\mathrm{O}(1)$ & $12(2)$ & $15(2)$ & $19(2)$ & $-1(1)$ & $4(1)$ & $3(1)$ \\
\hline
\end{tabular}


$\mathrm{La}\left(\mathrm{IO}_{3}\right)_{2} \mathrm{Cl}$

\begin{tabular}{lllllll}
\hline & $\mathrm{U} 11$ & $\mathrm{U} 22$ & $\mathrm{U} 33$ & $\mathrm{U} 23$ & $\mathrm{U} 13$ & $\mathrm{U} 12$ \\
\hline $\mathrm{La}(2)$ & $8(1)$ & $9(1)$ & $7(1)$ & $1(1)$ & $0(1)$ & $0(1)$ \\
$\mathrm{La}(1)$ & $7(1)$ & $8(1)$ & $7(1)$ & 0 & $0(1)$ & 0 \\
$\mathrm{I}(2)$ & $8(1)$ & $8(1)$ & $6(1)$ & $1(1)$ & $0(1)$ & $0(1)$ \\
$\mathrm{I}(3)$ & $8(1)$ & $8(1)$ & $6(1)$ & $0(1)$ & $-1(1)$ & $0(1)$ \\
$\mathrm{I}(1)$ & $9(1)$ & $8(1)$ & $7(1)$ & $-2(1)$ & $0(1)$ & $0(1)$ \\
$\mathrm{Cl}(1)$ & $23(1)$ & $16(1)$ & $11(1)$ & $2(1)$ & $4(1)$ & $2(1)$ \\
$\mathrm{Cl}(2)$ & $21(1)$ & $42(1)$ & $13(1)$ & $-11(1)$ & $0(1)$ & $-6(1)$ \\
$\mathrm{O}(2)$ & $13(2)$ & $10(2)$ & $13(2)$ & $-3(1)$ & $-2(1)$ & $-2(1)$ \\
$\mathrm{O}(6)$ & $14(2)$ & $14(2)$ & $6(2)$ & $5(1)$ & $0(1)$ & $-2(1)$ \\
$\mathrm{O}(1)$ & $20(2)$ & $15(2)$ & $7(2)$ & $1(1)$ & $0(1)$ & $1(2)$ \\
$\mathrm{O}(4)$ & $11(2)$ & $14(2)$ & $13(2)$ & $3(1)$ & $1(1)$ & $-3(1)$ \\
$\mathrm{O}(3)$ & $15(2)$ & $17(2)$ & $1(2)$ & $2(1)$ & $1(1)$ & $4(1)$ \\
$\mathrm{O}(9)$ & $11(2)$ & $20(2)$ & $26(3)$ & $-4(2)$ & $-7(2)$ & $-1(2)$ \\
$\mathrm{O}(7)$ & $10(2)$ & $15(2)$ & $21(2)$ & $-2(2)$ & $-3(2)$ & $1(1)$ \\
$\mathrm{O}(5)$ & $20(2)$ & $14(2)$ & $7(2)$ & $0(1)$ & $1(2)$ & $-6(1)$ \\
$\mathrm{O}(8)$ & $14(2)$ & $13(2)$ & $20(2)$ & $4(2)$ & $-1(2)$ & $7(1)$ \\
\hline & & & & & & \\
\hline
\end{tabular}

$\mathrm{La}_{2}\left(\mathrm{IO}_{3}\right)_{5} \mathrm{Cl}$

\begin{tabular}{lllllll}
\hline & $\mathrm{U}^{11}$ & $\mathrm{U}^{22}$ & $\mathrm{U}^{33}$ & $\mathrm{U}^{23}$ & $\mathrm{U}^{13}$ & $\mathrm{U}^{12}$ \\
\hline $\mathrm{La}(1)$ & $4(1)$ & $6(1)$ & $8(1)$ & $0(1)$ & $1(1)$ & $0(1)$ \\
$\mathrm{La}(2)$ & $4(1)$ & $8(1)$ & $7(1)$ & $0(1)$ & $2(1)$ & $1(1)$ \\
$\mathrm{I}(4)$ & $4(1)$ & $7(1)$ & $9(1)$ & $-1(1)$ & $2(1)$ & $1(1)$ \\
$\mathrm{I}(2)$ & $4(1)$ & $7(1)$ & $6(1)$ & $0(1)$ & $1(1)$ & $0(1)$ \\
$\mathrm{I}(5)$ & $5(1)$ & $10(1)$ & $9(1)$ & $-1(1)$ & $1(1)$ & $-1(1)$ \\
$\mathrm{I}(3)$ & $6(1)$ & $7(1)$ & $9(1)$ & $2(1)$ & $2(1)$ & $0(1)$ \\
$\mathrm{I}(1)$ & $7(1)$ & $8(1)$ & $7(1)$ & $0(1)$ & $2(1)$ & $0(1)$ \\
$\mathrm{Cl}(1)$ & $19(1)$ & $18(1)$ & $14(1)$ & $3(1)$ & $7(1)$ & $1(1)$ \\
$\mathrm{O}(7)$ & $8(2)$ & $10(2)$ & $11(2)$ & $2(2)$ & $-1(2)$ & $2(2)$ \\
$\mathrm{O}(12)$ & $17(2)$ & $19(3)$ & $8(2)$ & $1(2)$ & $-7(2)$ & $-2(2)$ \\
$\mathrm{O}(2)$ & $6(2)$ & $19(3)$ & $17(2)$ & $-1(2)$ & $3(2)$ & $1(2)$ \\
$\mathrm{O}(3)$ & $7(2)$ & $21(3)$ & $17(2)$ & $-3(2)$ & $1(2)$ & $-6(2)$ \\
$\mathrm{O}(14)$ & $9(2)$ & $14(2)$ & $12(2)$ & $-2(2)$ & $8(2)$ & $-4(2)$ \\
$\mathrm{O}(1)$ & $19(3)$ & $17(3)$ & $20(2)$ & $0(2)$ & $12(2)$ & $-2(2)$ \\
$\mathrm{O}(4)$ & $8(2)$ & $15(2)$ & $12(2)$ & $2(2)$ & $-2(2)$ & $-1(2)$ \\
$\mathrm{O}(6)$ & $19(3)$ & $9(2)$ & $20(2)$ & $6(2)$ & $2(2)$ & $4(2)$ \\
$\mathrm{O}(11)$ & $9(2)$ & $10(2)$ & $16(2)$ & $4(2)$ & $-2(2)$ & $0(2)$ \\
& & & & & & \\
\hline
\end{tabular}




\begin{tabular}{lllllll}
\hline $\mathrm{O}(8)$ & $5(2)$ & $7(2)$ & $17(2)$ & $-2(2)$ & $0(2)$ & $-3(2)$ \\
$\mathrm{O}(5)$ & $12(2)$ & $16(2)$ & $14(2)$ & $-2(2)$ & $4(2)$ & $0(2)$ \\
$\mathrm{O}(10)$ & $9(2)$ & $15(2)$ & $20(2)$ & $9(2)$ & $1(2)$ & $1(2)$ \\
$\mathrm{O}(9)$ & $7(2)$ & $17(2)$ & $8(2)$ & $3(2)$ & $3(2)$ & $-4(2)$ \\
$\mathrm{O}(15)$ & $17(2)$ & $9(2)$ & $24(3)$ & $-10(2)$ & $8(2)$ & $0(2)$ \\
$\mathrm{O}(13)$ & $15(2)$ & $19(3)$ & $19(2)$ & $-2(2)$ & $10(2)$ & $-7(2)$ \\
\hline
\end{tabular}

Table S3. The birefringence of metal iodates without other large polarizability anisotropy groups.

\begin{tabular}{|c|c|c|c|}
\hline Compound & $\begin{array}{l}\text { Crystallographically } \\
\text { independent I atom }\end{array}$ & Calculated birefringence & Reference \\
\hline $\mathrm{LiIO}_{3}$ & 1 & 0.146 at $1064 \mathrm{~nm}$ & 1 \\
\hline $\mathrm{RbIO}_{3}$ & 1 & 0.06 at & 2 \\
\hline $\mathrm{CsIO}_{3}$ & 1 & 0.19 at $1064 \mathrm{~nm}$ & 3 \\
\hline $\operatorname{LiMg}\left(\mathrm{IO}_{3}\right)_{3}$ & 1 & 0.22 at $1064 \mathrm{~nm}$ & 4 \\
\hline $\operatorname{LiZn}\left(\mathrm{IO}_{3}\right)_{3}$ & 1 & 0.27 at $1064 \mathrm{~nm}$ & 5 \\
\hline $\mathrm{LiCd}\left(\mathrm{IO}_{3}\right)_{3}$ & 1 & 0.27 at $1064 \mathrm{~nm}$ & 5 \\
\hline $\mathrm{Li}_{2} \mathrm{Ge}\left(\mathrm{IO}_{3}\right)_{6}$ & 1 & 0.21 at $1064 \mathrm{~nm}$ & 6 \\
\hline$\alpha-\left(\mathrm{H}_{3} \mathrm{O}\right)_{2} \mathrm{Pt}\left(\mathrm{IO}_{3}\right)_{6}$ & 1 & 0.28 at $2000 \mathrm{~nm}$ & 7 \\
\hline $\mathrm{Ba}_{3} \mathrm{Ga}_{2}\left(\mathrm{IO}_{3}\right)_{12}$ & 1 & 0.103 at $1064 \mathrm{~nm}$ & 8 \\
\hline $\mathrm{ZnIO}_{3} \mathrm{~F}$ & 1 & 0.219 at $546 \mathrm{~nm}$ & 9 \\
\hline $\mathrm{Bi}\left(\mathrm{IO}_{3}\right) \mathrm{F}_{2}$ & 1 & 0.209 at $1064 \mathrm{~nm}$ & 10 \\
\hline $\mathrm{Ba}\left(\mathrm{IO}_{3}\right) \mathrm{Cl}$ & 1 & 0.118 at $1064 \mathrm{~nm}$ & This work \\
\hline $\mathrm{Ba}\left(\mathrm{IO}_{3}\right) \mathrm{Cl}\left(\mathrm{H}_{2} \mathrm{O}\right)$ & 1 & 0.106 at $1064 \mathrm{~nm}$ & This work \\
\hline $\mathrm{BaGe}\left(\mathrm{IO}_{3}\right)_{6} \cdot \mathrm{H}_{2} \mathrm{O}$ & 2 & 0.02 at $1064 \mathrm{~nm}$ & 6 \\
\hline $\mathrm{Ce}\left(\mathrm{IO}_{3}\right)_{2} \mathrm{~F}_{2} \cdot \mathrm{H}_{2} \mathrm{O}$ & 2 & 0.046 at $1064 \mathrm{~nm}$ & 11 \\
\hline $\mathrm{Li}_{2} \mathrm{Ce}\left(\mathrm{IO}_{3}\right)_{4} \mathrm{~F}_{2}$ & 2 & 0.054 at $589 \mathrm{~nm}$ & 12 \\
\hline $\mathrm{Bi}_{3} \mathrm{OF}_{3}\left(\mathrm{IO}_{3}\right)_{4}$ & 2 & 0.057 at $532 \mathrm{~nm}$ & 13 \\
\hline$\left(\mathrm{NH}_{4}\right) \mathrm{Bi}_{2}\left(\mathrm{IO}_{3}\right)_{2} \mathrm{~F}_{5}$ & 2 & 0.0690 at $589.3 \mathrm{~nm}$ & 14 \\
\hline $\mathrm{Sn}\left(\mathrm{IO}_{3}\right)_{2} \mathrm{~F}_{2}$ & 2 & 0.234 at $1064 \mathrm{~nm}$ & 15 \\
\hline $\mathrm{Ba}_{7}\left(\mathrm{IO}_{3}\right)_{5} \mathrm{Cl}_{9}$ & 2 & 0.023 at $1064 \mathrm{~nm}$ & This work \\
\hline $\mathrm{K}_{2} \mathrm{Bi}\left(\mathrm{IO}_{3}\right)_{5}$ & 3 & 0.0536 at $1064 \mathrm{~nm}$ & 16 \\
\hline
\end{tabular}




\begin{tabular}{|c|c|c|c|}
\hline $\mathrm{Rb}_{2} \mathrm{Bi}\left(\mathrm{IO}_{3}\right)_{5}$ & 3 & 0.0427 at $1064 \mathrm{~nm}$ & 16 \\
\hline $\mathrm{Sr}\left(\mathrm{IO}_{3}\right)_{3} \mathrm{H}$ & 3 & 0.07 at $1064 \mathrm{~nm}$ & 17 \\
\hline $\mathrm{La}\left(\mathrm{IO}_{3}\right)_{2} \mathrm{Cl}$ & 3 & 0.038 at $1064 \mathrm{~nm}$ & This work \\
\hline $\mathrm{NaBi}\left(\mathrm{IO}_{3}\right)_{4}$ & 5 & 0.033 at $1064 \mathrm{~nm}$ & 18 \\
\hline $\mathrm{La}_{2}\left(\mathrm{IO}_{3}\right)_{5} \mathrm{Cl}$ & 5 & 0.045 at $1064 \mathrm{~nm}$ & This work \\
\hline
\end{tabular}




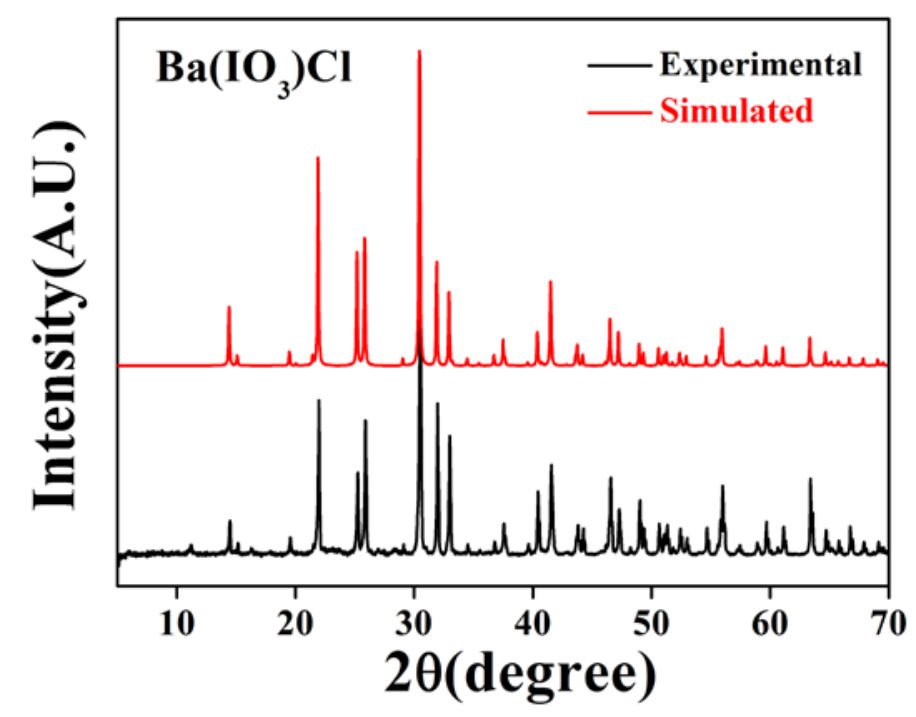

(a)

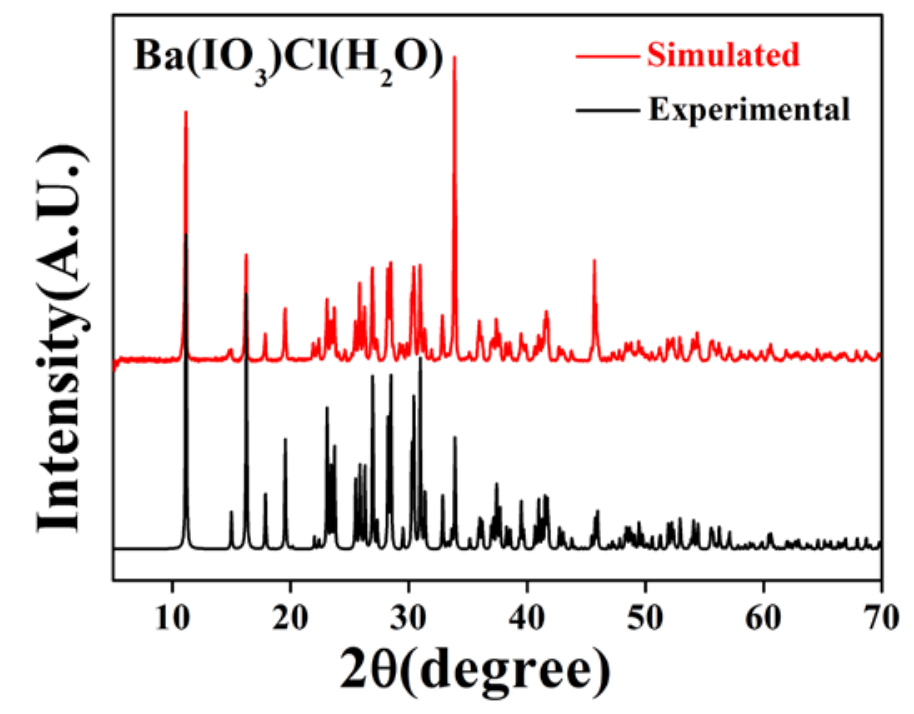

(b)

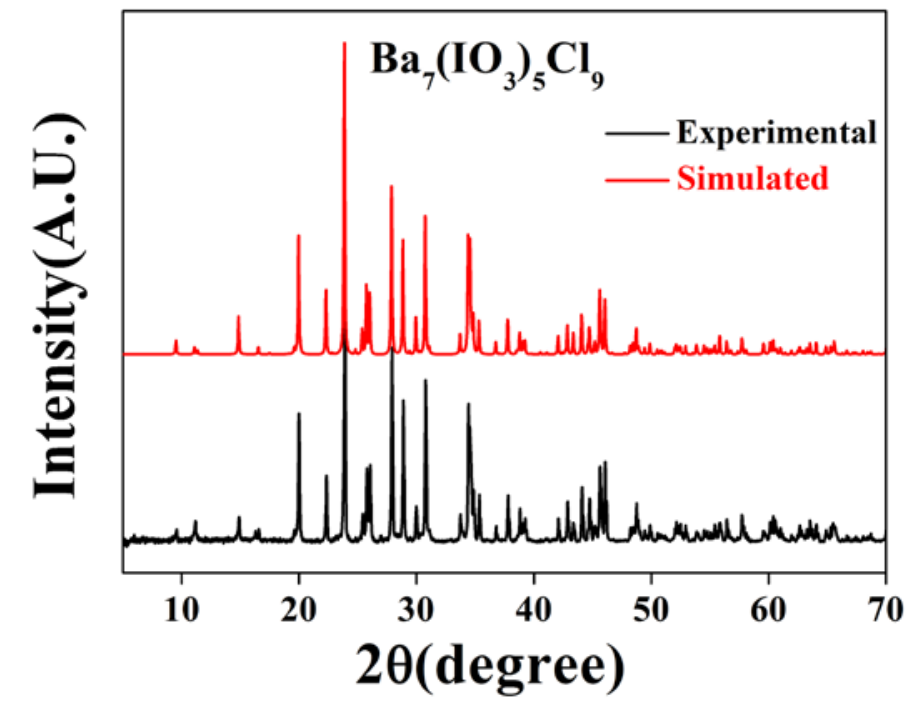

(c) 


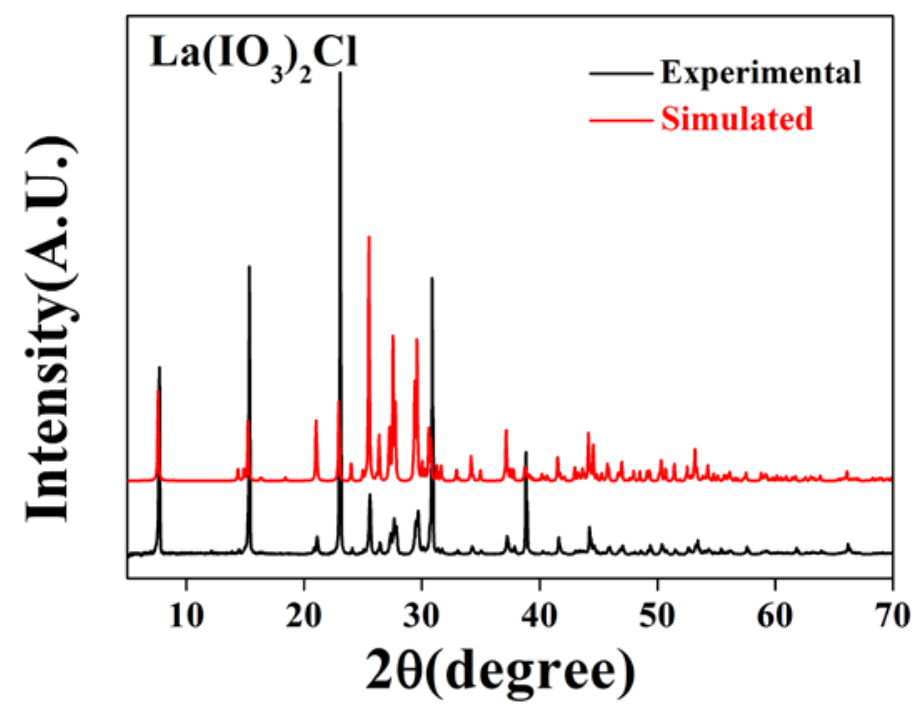

(d)

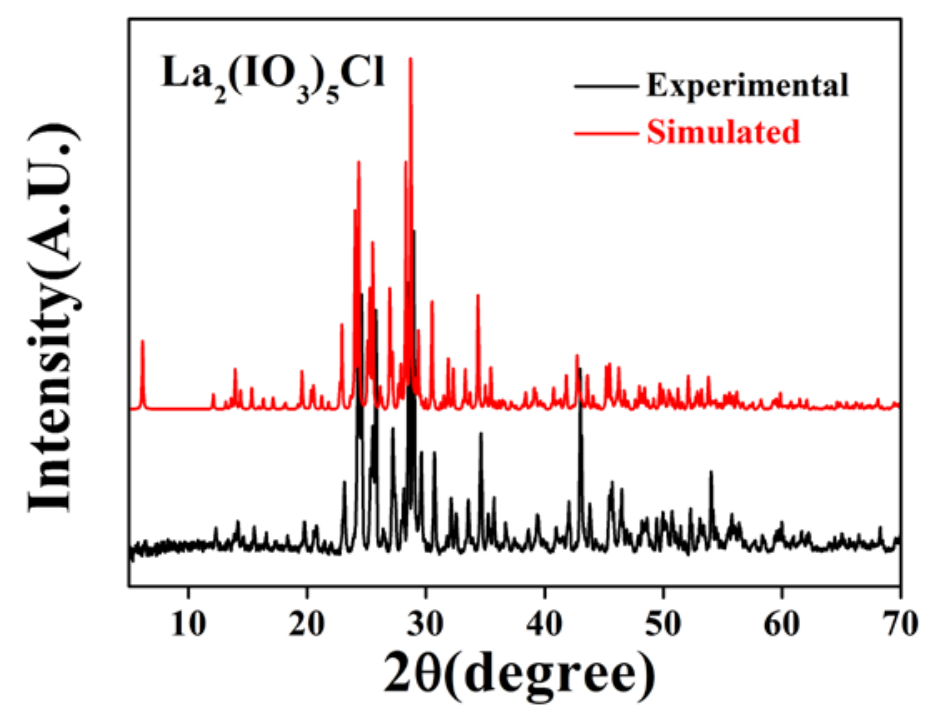

(e)

Figure S1. Experimental and simulated PXRD patterns of $\mathrm{Ba}\left(\mathrm{IO}_{3}\right) \mathrm{Cl}$ (a), $\mathrm{Ba}\left(\mathrm{IO}_{3}\right) \mathrm{Cl}\left(\mathrm{H}_{2} \mathrm{O}\right)(\mathbf{b}), \mathrm{Ba}_{7}\left(\mathrm{IO}_{3}\right)_{5} \mathrm{Cl}_{9}(\mathbf{c}), \mathrm{La}\left(\mathrm{IO}_{3}\right)_{2} \mathrm{Cl}(\mathbf{d})$ and $\mathrm{La}_{2}\left(\mathrm{IO}_{3}\right)_{5} \mathrm{Cl}(\mathbf{e})$.

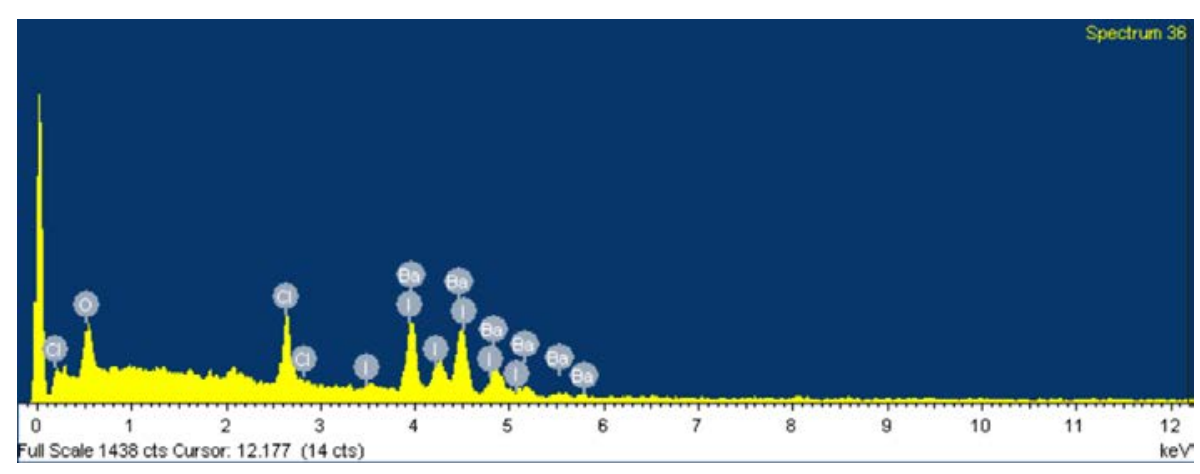

(a) 


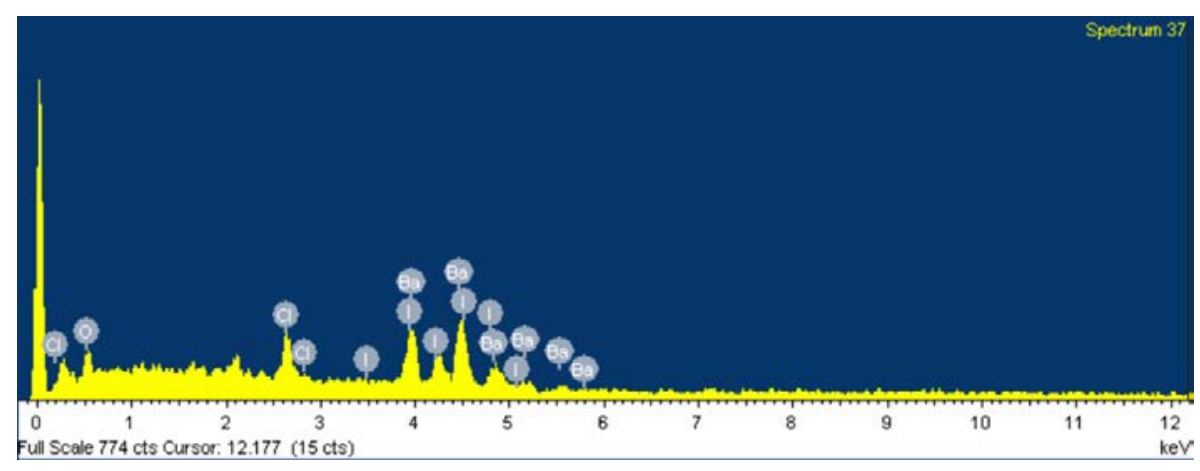

(b)

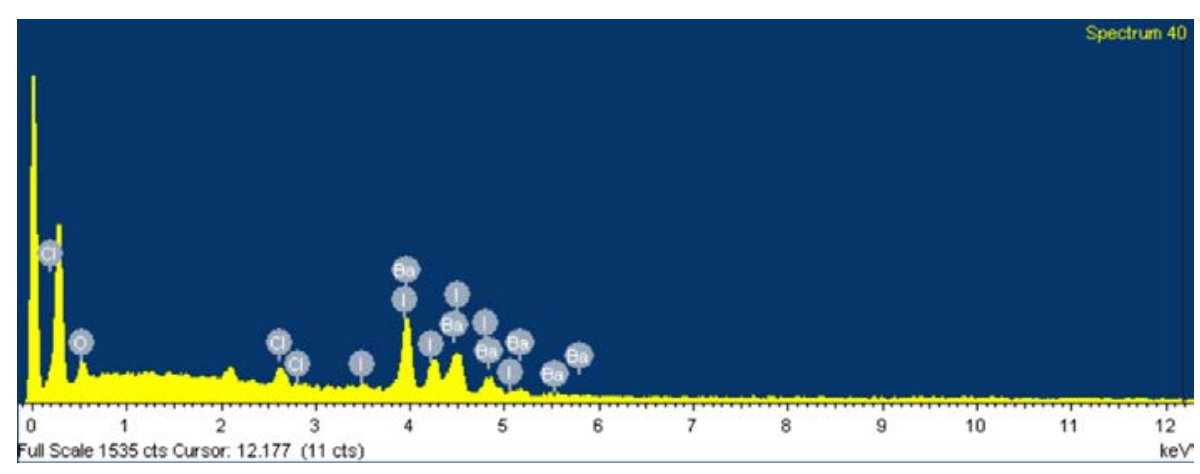

(c)

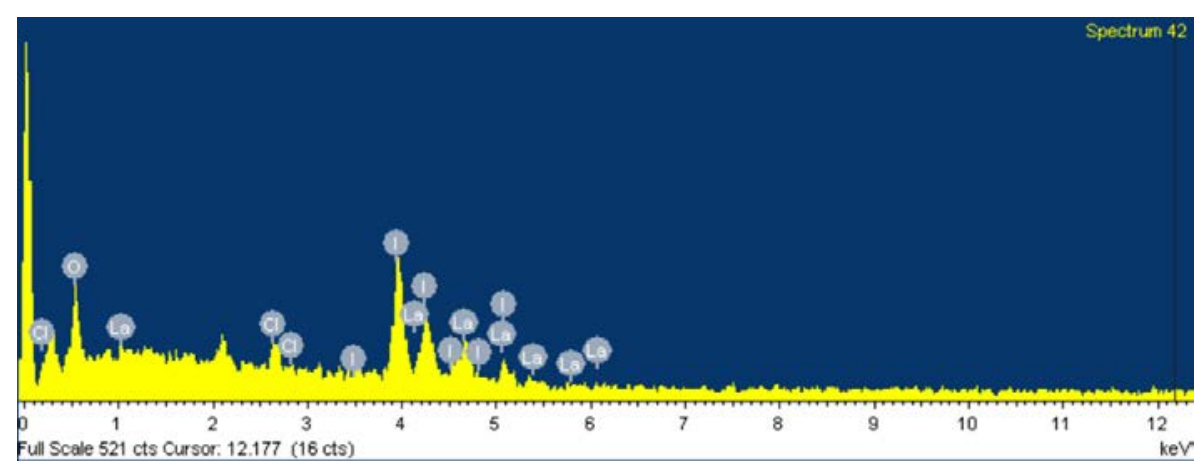

(d)

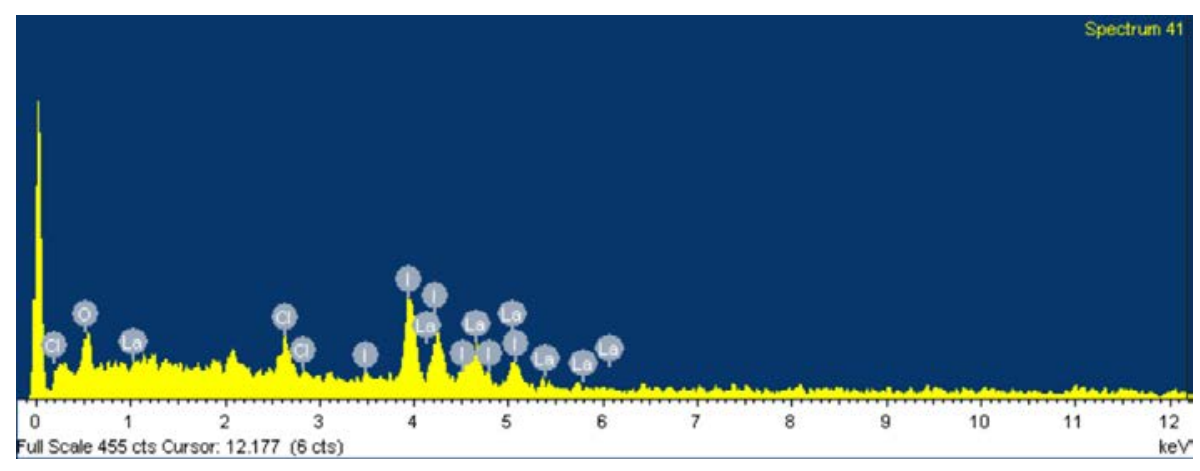

(e)

Figure S2. Energy-dispersive spectrometry (EDS) plots of $\mathrm{Ba}\left(\mathrm{IO}_{3}\right) \mathrm{Cl}$ (a), $\mathrm{Ba}\left(\mathrm{IO}_{3}\right) \mathrm{Cl}\left(\mathrm{H}_{2} \mathrm{O}\right)(\mathbf{b}), \mathrm{Ba}_{7}\left(\mathrm{IO}_{3}\right)_{5} \mathrm{Cl}_{9}(\mathbf{c}), \mathrm{La}\left(\mathrm{IO}_{3}\right)_{2} \mathrm{Cl}$ (d) and $\mathrm{La}_{2}\left(\mathrm{IO}_{3}\right)_{5} \mathrm{Cl}$ (e). 


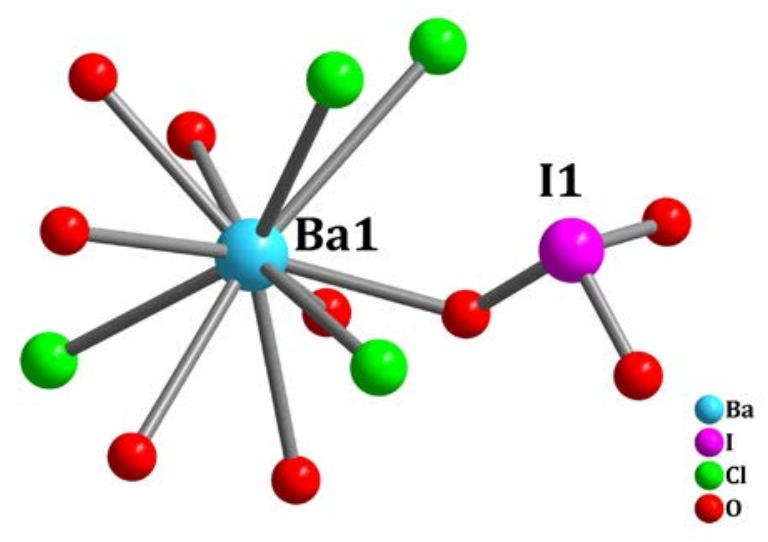

Figure S3. Asymmetric unit of $\mathrm{Ba}\left(\mathrm{IO}_{3}\right) \mathrm{Cl}$.

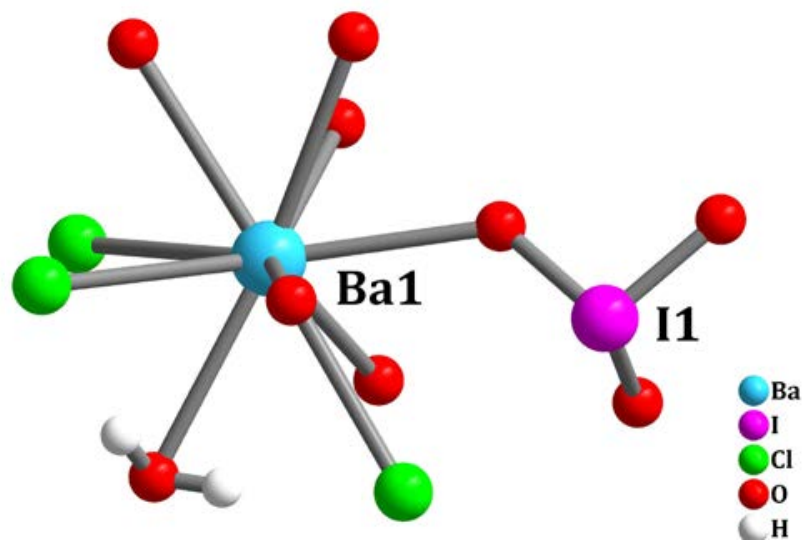

Figure S4. Asymmetric unit of $\mathrm{Ba}\left(\mathrm{IO}_{3}\right) \mathrm{Cl}\left(\mathrm{H}_{2} \mathrm{O}\right)$.

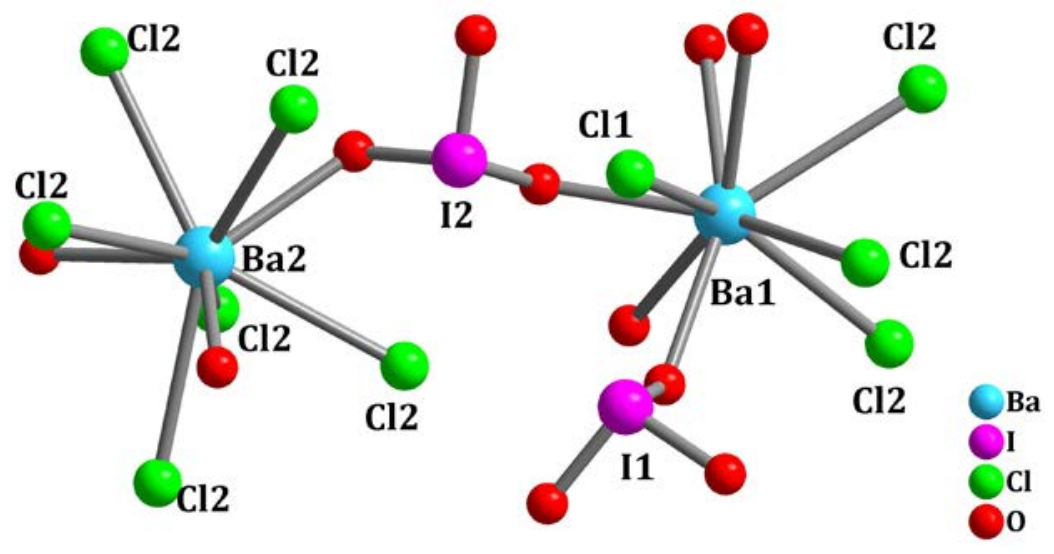

Figure S5. Asymmetric unit of $\mathrm{Ba}_{7}\left(\mathrm{IO}_{3}\right)_{5} \mathrm{Cl}_{9}$. 


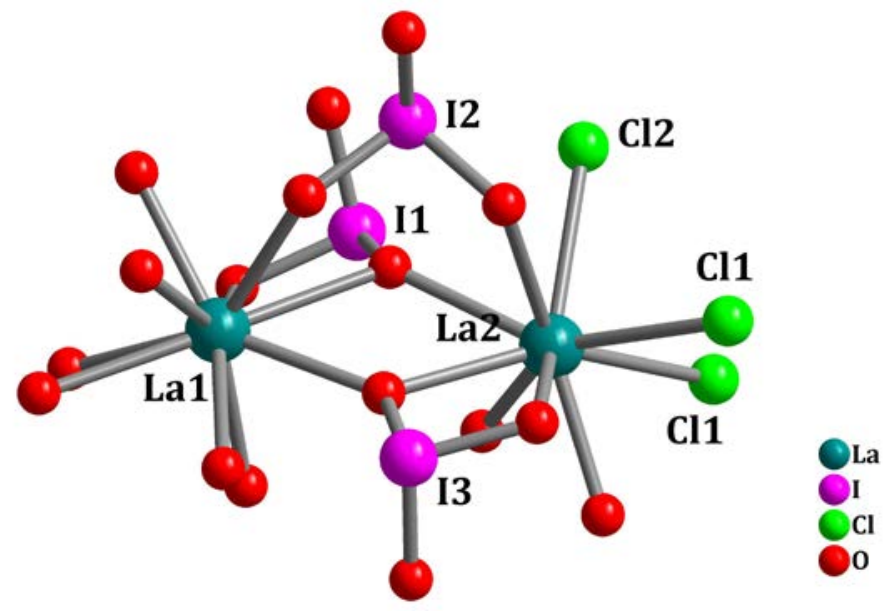

(b)

Figure S6. Asymmetric unit of $\mathrm{La}\left(\mathrm{IO}_{3}\right)_{2} \mathrm{Cl}$.

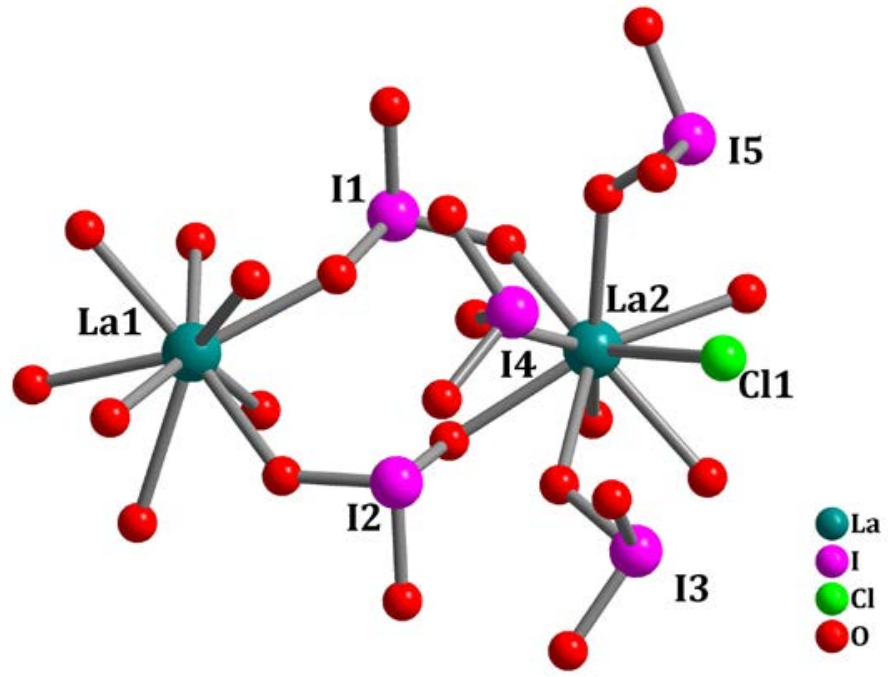

Figure S7. Asymmetric unit of $\mathrm{La}_{2}\left(\mathrm{IO}_{3}\right)_{5} \mathrm{Cl}$. 


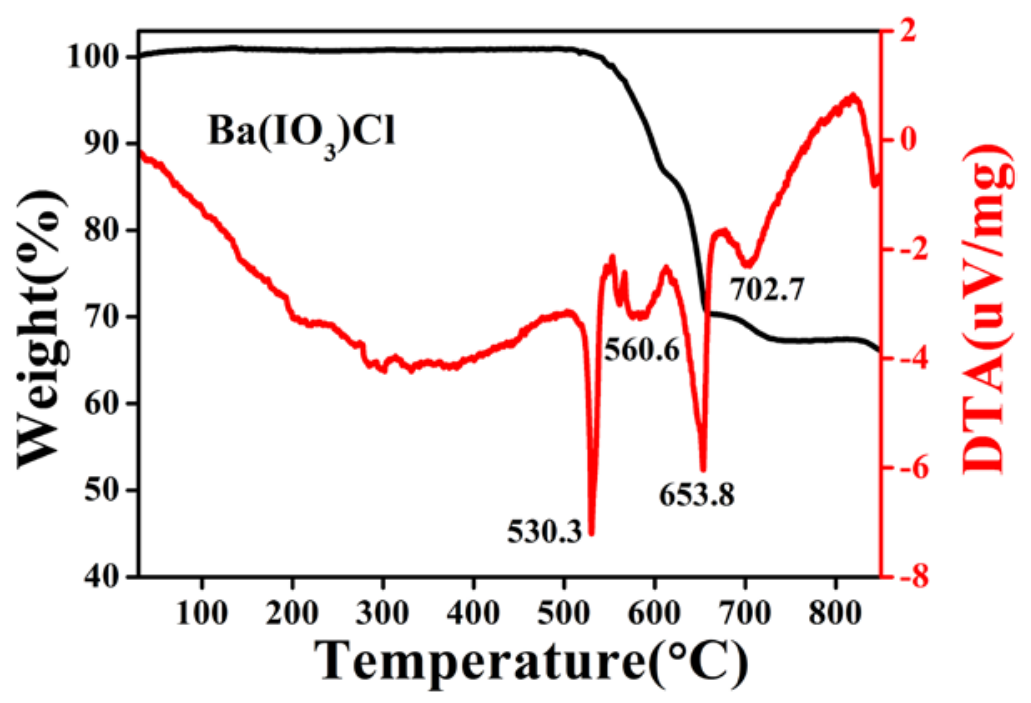

(a)

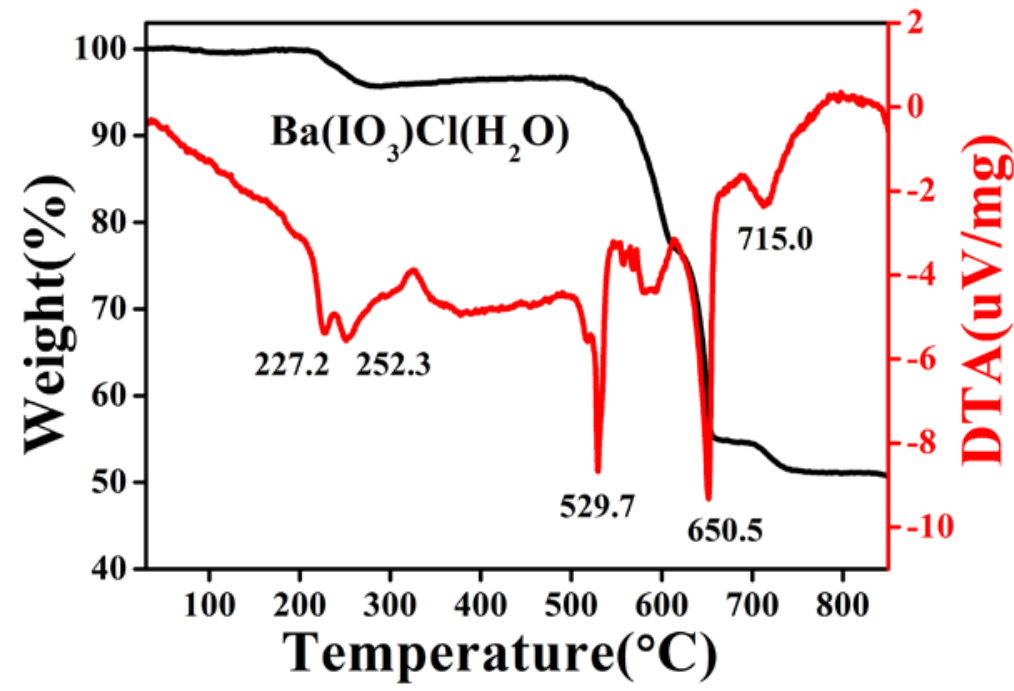

(b)

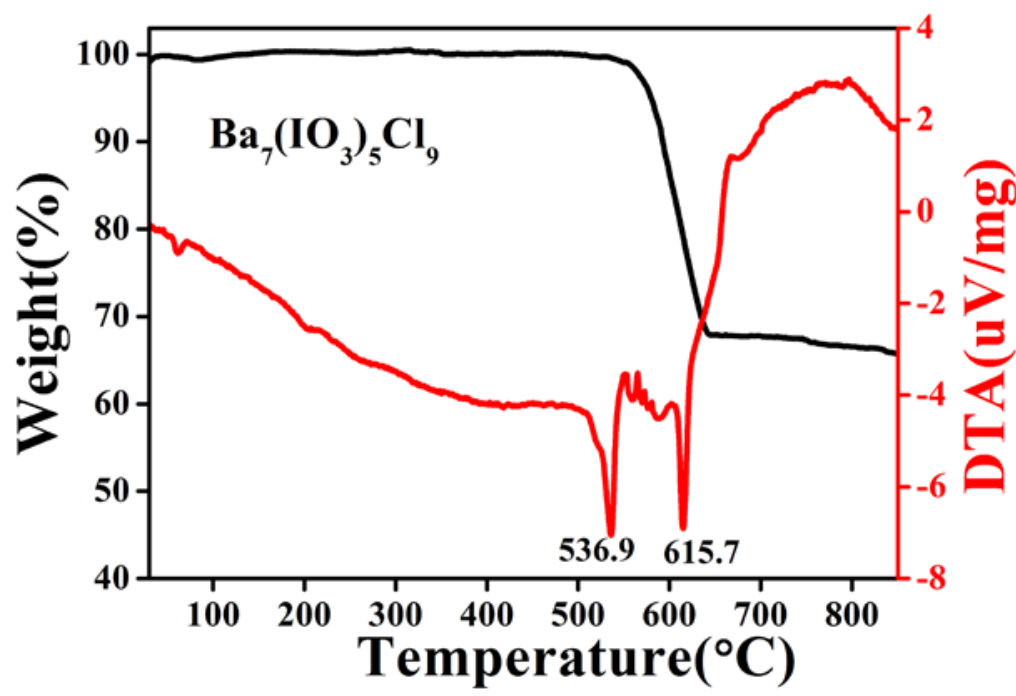

(c) 


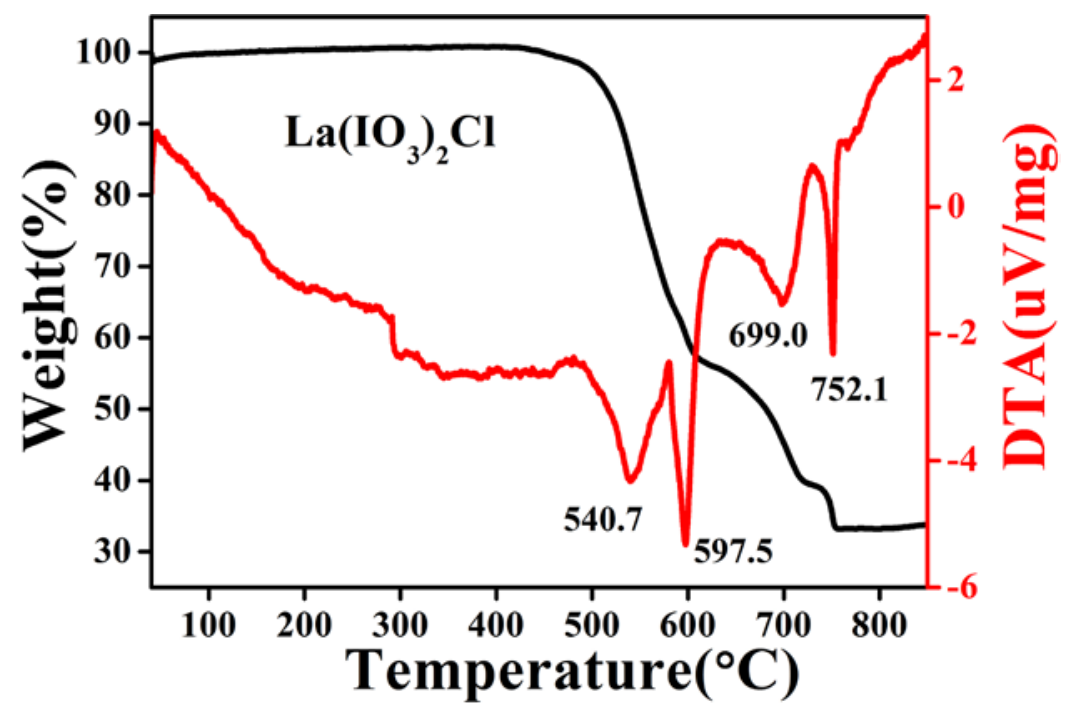

(d)

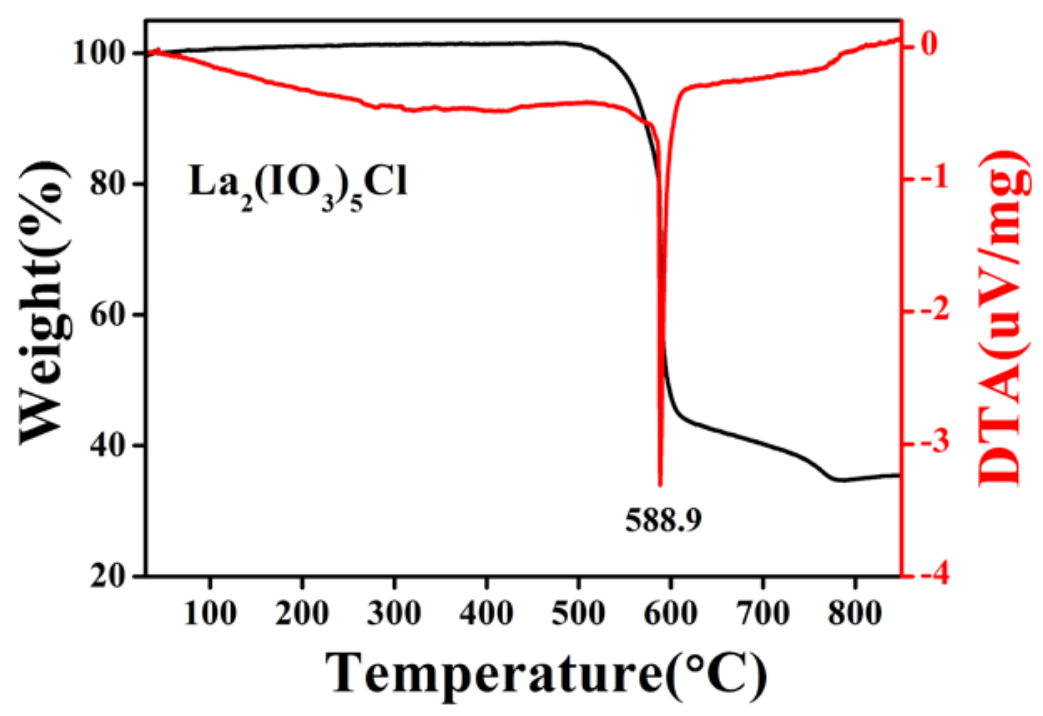

(e)

Figure S8. TG-DTA curves of $\mathrm{Ba}\left(\mathrm{IO}_{3}\right) \mathrm{Cl}(\mathbf{a}), \mathrm{Ba}\left(\mathrm{IO}_{3}\right) \mathrm{Cl}\left(\mathrm{H}_{2} \mathrm{O}\right)(\mathbf{b}), \mathrm{Ba}_{7}\left(\mathrm{IO}_{3}\right)_{5} \mathrm{Cl}_{9}$ (c), $\mathrm{La}\left(\mathrm{IO}_{3}\right)_{2} \mathrm{Cl}(\mathbf{d})$ and $\mathrm{La}_{2}\left(\mathrm{IO}_{3}\right)_{5} \mathrm{Cl}(\mathbf{e})$. 


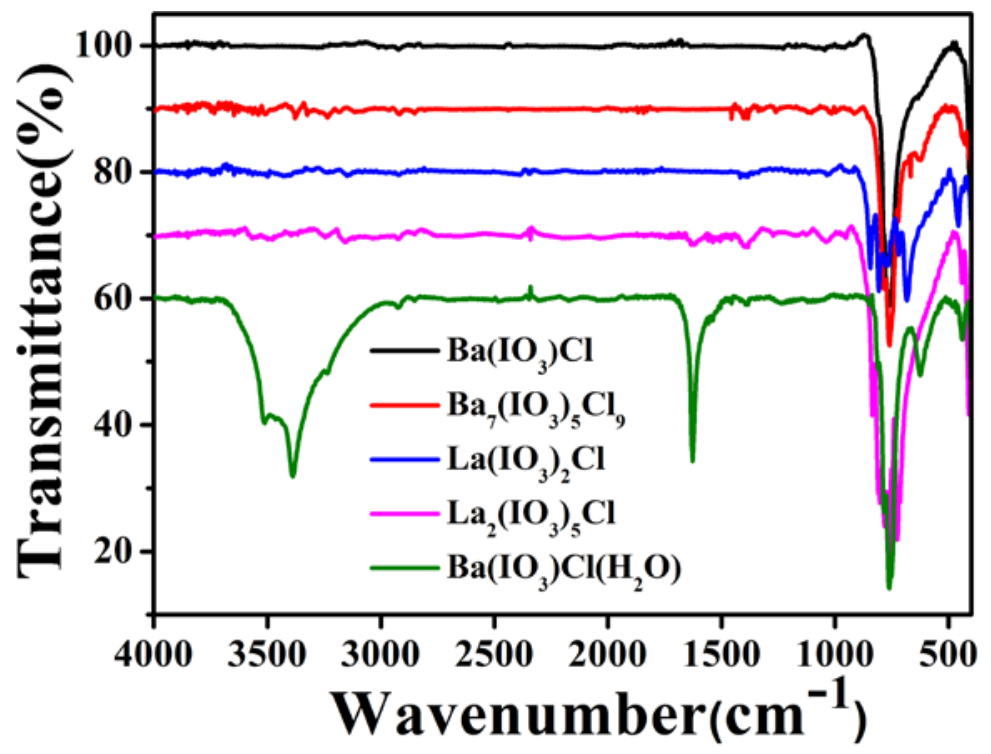

Figure S9. The IR spectra of $\mathrm{Ba}\left(\mathrm{IO}_{3}\right) \mathrm{Cl}, \mathrm{Ba}\left(\mathrm{IO}_{3}\right) \mathrm{Cl}\left(\mathrm{H}_{2} \mathrm{O}\right), \mathrm{Ba}_{7}\left(\mathrm{IO}_{3}\right)_{5} \mathrm{Cl}_{9}, \mathrm{La}\left(\mathrm{IO}_{3}\right)_{2} \mathrm{Cl}$ and $\mathrm{La}_{2}\left(\mathrm{IO}_{3}\right)_{5} \mathrm{Cl}$.

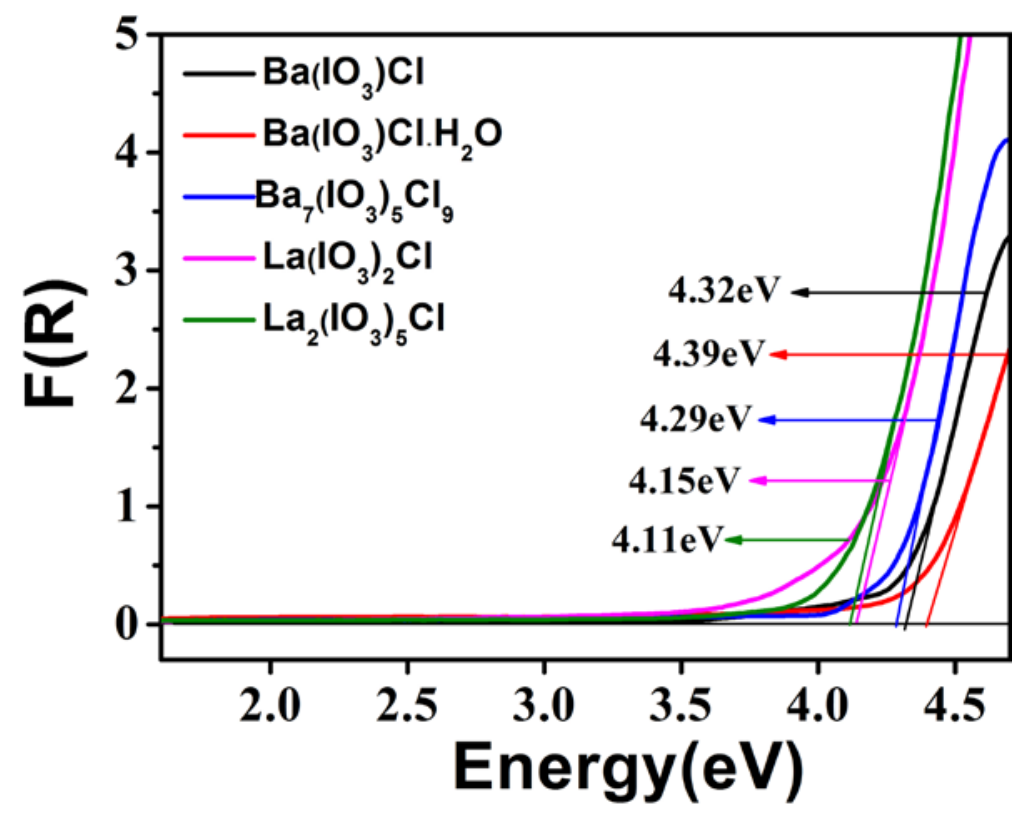

Figure S10. UV-vis-IR spectra of $\mathrm{Ba}\left(\mathrm{IO}_{3}\right) \mathrm{Cl}, \mathrm{Ba}\left(\mathrm{IO}_{3}\right) \mathrm{Cl}\left(\mathrm{H}_{2} \mathrm{O}\right), \mathrm{Ba}_{7}\left(\mathrm{IO}_{3}\right)_{5} \mathrm{Cl}_{9}$, $\mathrm{La}\left(\mathrm{IO}_{3}\right)_{2} \mathrm{Cl}$ and $\mathrm{La}_{2}\left(\mathrm{IO}_{3}\right)_{5} \mathrm{Cl}$. 

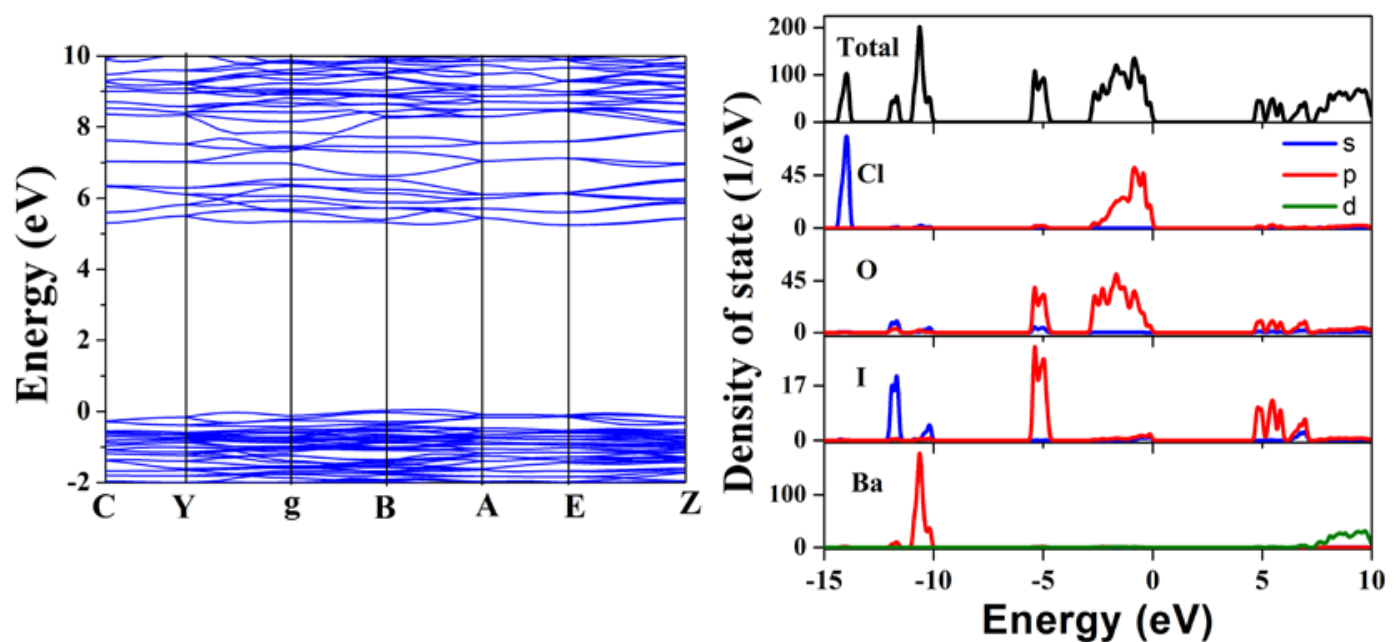

(a)
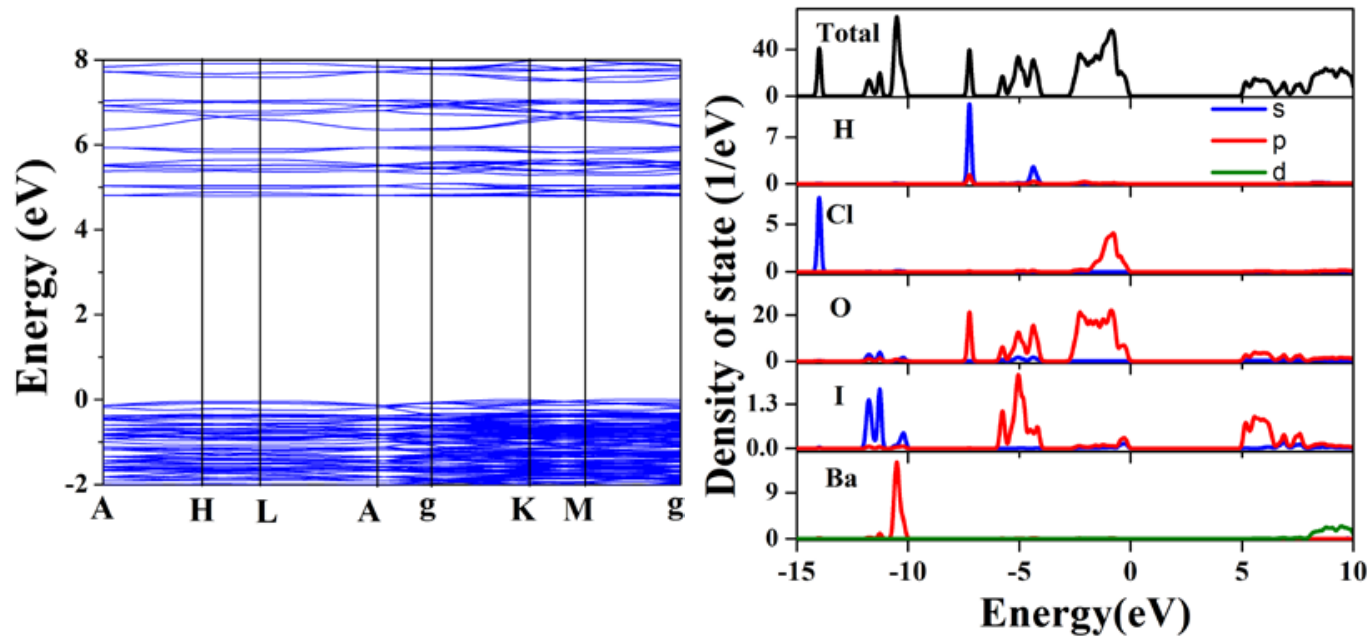

(b)
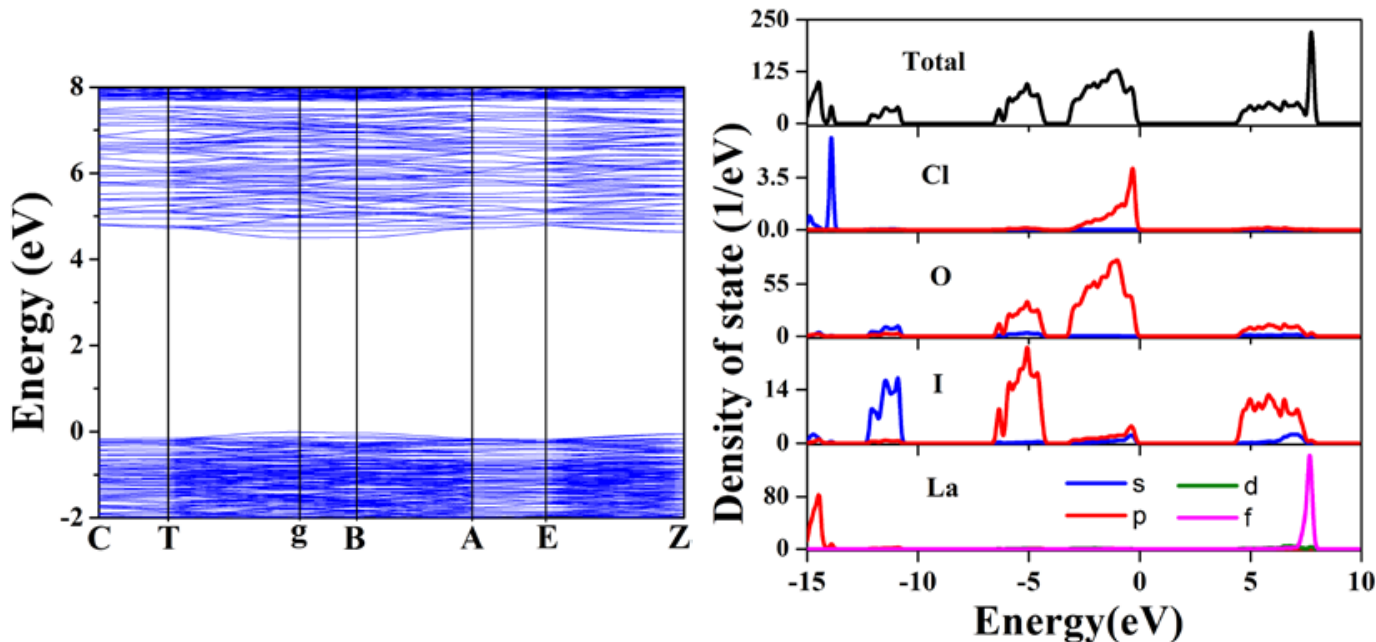

(c)

Figure S11. Calculated band structures, total and partial density of states (DOSs) of $\mathrm{Ba}\left(\mathrm{IO}_{3}\right) \mathrm{Cl}\left(\mathrm{H}_{2} \mathrm{O}\right)(\mathbf{a}), \mathrm{Ba}_{7}\left(\mathrm{IO}_{3}\right)_{5} \mathrm{Cl}_{9}(\mathbf{b})$ and $\mathrm{La}_{2}\left(\mathrm{IO}_{3}\right)_{5} \mathrm{Cl}(\mathbf{c})$. 


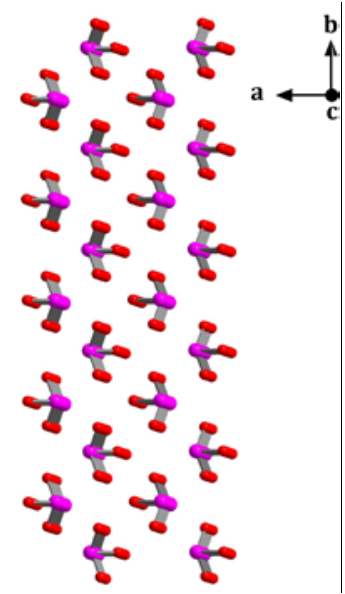

(a)

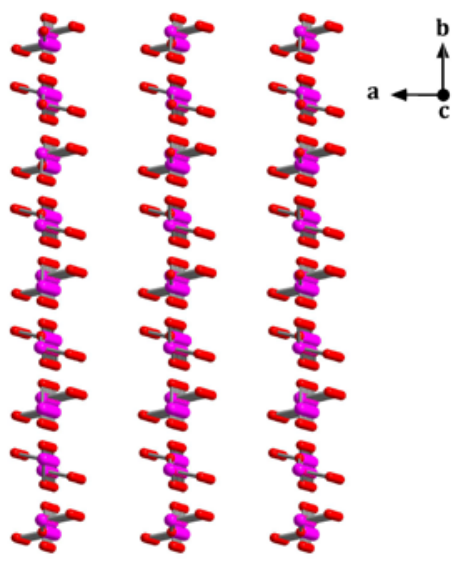

(b)

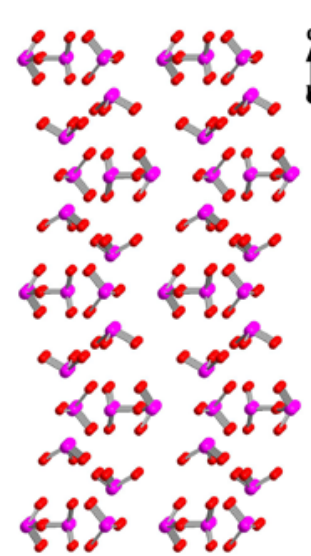

(c)

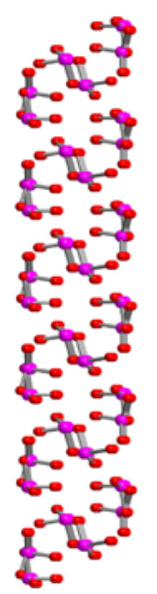

(d)

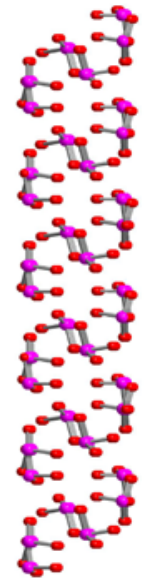

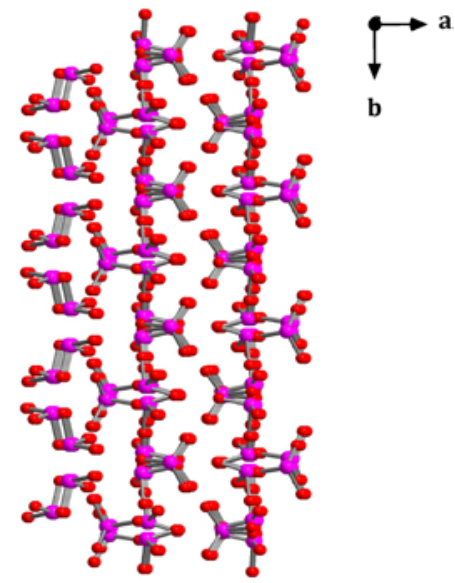

(e)

Figure S12. Distinct arrangement of the $\mathrm{IO}_{3}$ groups in $\mathrm{Ba}\left(\mathrm{IO}_{3}\right) \mathrm{Cl}$ (a), $\mathrm{Ba}\left(\mathrm{IO}_{3}\right) \mathrm{Cl}\left(\mathrm{H}_{2} \mathrm{O}\right)(\mathbf{b}), \mathrm{Ba}_{7}\left(\mathrm{IO}_{3}\right)_{5} \mathrm{Cl}_{9}(\mathbf{c}), \mathrm{La}\left(\mathrm{IO}_{3}\right)_{2} \mathrm{Cl}(\mathbf{d})$ and $\mathrm{La}_{2}\left(\mathrm{IO}_{3}\right)_{5} \mathrm{Cl}(\mathbf{e})$. 


\section{Reference}

1. V. G. Dmitriev, G. G. Gurzadyan and D. N. Nikogosyan, Handbook of Nonlinear Optical Crystals (Springer Series in Optical Sciences), vol. 64. A. E. Siegman, Springer Verlag, 1991.

2. Wu, Q.; Liu, H.; Jiang, F.; Kang, L.; Yang, L.; Lin, Z.; Hu, Z.; Chen, X.; Meng, X.; Qin, J. $\mathrm{RbIO}_{3}$ and $\mathrm{RbIO}_{2} \mathrm{~F}_{2}$ : Two Promising Nonlinear Optical Materials in Mid-IR Region and Influence of Partially Replacing Oxygen with Fluorine for Improving Laser Damage Threshold. Chem. Mater. 2016, 28, 1413-1418.

3. Zhang, M.; Hu, C.; Abudouwufu, T.; Yang, Z.; Pan, S. Functional Materials Design via Structural Regulation Originated from Ions Introduction: A Study Case in Cesium Iodate System. Chem. Mater. 2018, 30, 1136-1145.

4. Chen, J.; Hu, C.-L.; Mao, F. F.; Zhang, X.-H.; Yang, B.-P.; Mao, J.-G. LiMg $\left(\mathrm{IO}_{3}\right)_{3}$ : An excellent SHG material designed by single-site aliovalent substitution. Chem. Sci., 2019, 10, 10870-10875.

5. Jia, Y.-J.; Chen, Y.-G.; Guo, Y.; Guan, X.-F.; Li, C.; Li, B.; Liu, M.-M.; Zhang, X.-M., $\operatorname{LiM}^{\mathrm{II}}\left(\mathrm{IO}_{3}\right)_{3}\left(\mathrm{M}^{\mathrm{II}}=\mathrm{Zn}\right.$ and Cd): Two Promising Nonlinear Optical Crystals Derived from a Tunable Structure Model of $\alpha-\mathrm{LiIO}_{3}$. Angew. Chem., Int. Ed. 2019, 58, 17194-17198.

6. Liu, H.; Jiang, X.; Wang, X.; Yang, L.; Lin, Z.; Hu, Z.; Meng, X.; Chen, X.; Qin, J. Influence of A-site cations on germanium iodates as mid-IR nonlinear optical materials: $\mathrm{A}_{2} \mathrm{Ge}\left(\mathrm{IO}_{3}\right)_{6}(\mathrm{~A}=\mathrm{Li}, \mathrm{K}, \mathrm{Rb}$ and $\mathrm{Cs})$ and $\mathrm{BaGe}\left(\mathrm{IO}_{3}\right)_{6} \cdot \mathrm{H}_{2} \mathrm{O}$. J. Mater. Chem. C., 2018, 6, 4698-4705.

7. Yang, B. P.; Hu, C. L.; Xu, X.; Mao, J. G. New Series of Polar and Nonpolar Platinum Iodates $\mathrm{A}_{2} \mathrm{Pt}\left(\mathrm{IO}_{3}\right)_{6}\left(\mathrm{~A}=\mathrm{H}_{3} \mathrm{O}, \mathrm{Na}, \mathrm{K}, \mathrm{Rb}, \mathrm{Cs}\right)$. Inorg. Chem. 2016, 55, $2481-2487$.

8. Xiao, L.; You, F.; Gong, P.; Hu, Z.; Lin, Z. Synthesis and structure of a new mixed metal iodate $\mathrm{Ba}_{3} \mathrm{Ga}_{2}\left(\mathrm{IO}_{3}\right)_{12}$. CrystEngComm. 2019, 21, 4981-4986.

9. Gai, M.; Wang, Y.; Tong, T.; Yang, Z.; Pan, S. ZnIO 3 F: Zinc Iodate Fluoride with Large Birefringence and Wide Band Gap. Inorg. Chem. 2020, 59, 4172-4175. 
10. Mao, F. F.; Hu, C. L.; Xu, X.; Yan, D.; Yang, B. P.; Mao, J. G. Bi( $\left(\mathrm{IO}_{3}\right) \mathrm{F}_{2}$ : The first metal iodate fluoride with a very strong second harmonic generation effect. Angew. Chem., Int. Ed. 2017, 56 (8), 2151-2155.

11. Abudouwufu, T.; Zhang, M.; Cheng, S.; Yang, Z.; Pan, S. Ce( $\left(\mathrm{IO}_{3}\right)_{2} \mathrm{~F}_{2} \cdot \mathrm{H}_{2} \mathrm{O}$ : The First Rare Earth Iodate Fluoride with Large Second Harmonic Generation Response. Chem. - Eur. J. 2018, 25, 1221-1226.

12. Tang, C.; Jiang, X.; Guo, S.; Guo, R.; Liu, L.; Xia, M.; Huang, Q.; Lin, Z.; Wang, X. Synthesis, Crystal Structure, and Optical Properties of the First Alkali Metal Rare-Earth Iodate Fluoride: $\mathrm{Li}_{2} \mathrm{Ce}\left(\mathrm{IO}_{3}\right)_{4} \mathrm{~F}_{2}$. Cryst. Growth \& Des. 2020, 20, $2135-2140$.

13. Zhang, M.; Su, X.; Mutailipu, M.; Yang, Z. H.; Pan, S. L. $\mathrm{Bi}_{3} \mathrm{OF}_{3}\left(\mathrm{IO}_{3}\right)_{4}$ : Metal oxyiodate fluoride featuring a carbon-nanotube-like topological structure with large second harmonic generation response. Chem. Mater. 2017, 29 (3), 945-949.

14. Fan, H. X.; Lin, C. S.; Chen, K. C.; Peng, G.; Li, B. X.; Zhang, G.; Long, X. F.; Ye, N. $\left(\mathrm{NH}_{4}\right) \mathrm{Bi}_{2}\left(\mathrm{IO}_{3}\right)_{3} \mathrm{~F}_{5}$ : An unusual ammonium-containing metal iodate fluoride showing strong second harmonic generation (SHG) response and thermochromic behavior. Angew. Chem., Int. Ed. 2020, 59, 5268-5272.

15. Luo, M.; Liang, F.; Hao, X.; Lin, D.; Li, B.; Lin, Z.; Ye, N. Rational Design of the Nonlinear Optical Response in a Tin Iodate Fluoride $\mathrm{Sn}\left(\mathrm{IO}_{3}\right)_{2} \mathrm{~F}_{2}$. Chem. Mater. 2020, 32 (6), 2615-2620.

16. Huang, Y.; Meng, X.; Gong, P.; Yang, L.; Lin, Z.; Chen, X.; Qin, J. $\mathrm{A}_{2} \mathrm{BiI}_{5} \mathrm{O}_{15}$ (A $=\mathrm{K}^{+}$or $\mathrm{Rb}^{+}$): two new promising nonlinear optical materials containing $\left[\mathrm{I}_{3} \mathrm{O}_{9}\right]^{3-}$ bridging anionic groups. J. Mater. Chem. C., 2014, 2, 4057-4062.

17. Song, H.; Wang, N.; Li, Y.; Liu, W.; Lin, Z.; Yao, J.; Zhang, G. $\operatorname{SrI}_{3} \mathrm{O}_{9} H$ : A new alkaline earth metal iodate with two different anionic units using mild aqua-solution method. Solid State Sci. 2019, 97, 105982.

18. Jia, Y.-J.; Chen, Y.-G.; Wang, T.; Guo, Y.; Guan, X.-F.; Zhang, X.-M. $\mathrm{KBi}\left(\mathrm{IO}_{3}\right)_{3}(\mathrm{OH})$ and $\mathrm{NaBi}\left(\mathrm{IO}_{3}\right)_{4}$ : from the centrosymmetric chain to a noncentrosymmetric double layer. Dalton Trans. 2019, 48, 10320-10326. 